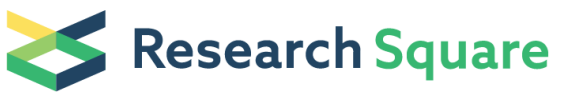 \\ Preprints are preliminary reports that have not undergone peer review. \\ They should not be considered conclusive, used to inform clinical practice, or referenced by the media as validated information.
}

\section{Improved Strategy for Jet-in-air Cell Sorting With High Purity, Yield, Viability and Genome Stability}

\section{Xinghui Song}

Zhejiang University School of Medicine

\section{Jiajia Wang}

Zhejiang University School of Medicine

\section{Yanwei Li}

Zhejiang University School of Medicine

\section{Yueting Xing}

Zhejiang University School of Medicine

\section{Chun Guo}

Zhejiang University School of Medicine

\section{Yingying Huang}

Zhejiang University School of Medicine

\section{Lintao Xu}

Zhejiang University School of Medicine Second Affiliated Hospital

\section{$\mathrm{Hu} \mathrm{Hu}$}

Zhejiang University School of Medicine

\section{LINLIN WANG ( $\nabla$ wanglinlin@zju.edu.cn )}

Department of Basic Medicine Sciences, and Department of Orthopaedics of Sir Run Run Shaw Hospital, Zhejiang University School of Medicine, Hangzhou, Zhejiang, China https://orcid.org/0000-0002-7720-3012

\section{Methodology}

Keywords: jet-in-air cell sorting, single-cell sequencing, drop delay, yield, viability, cell counts, gene expression

Posted Date: December 16th, 2020

DOI: https://doi.org/10.21203/rs.3.rs-127241/v1

License: (1) This work is licensed under a Creative Commons Attribution 4.0 International License. Read Full License 


\section{Abstract}

Background: The enrichment of viable cells is an essential step in single-cell sequencing for clinical and biotechnology research applications. A high-end jet-in-air sorter is a fluorescent-activated cell sorting sorter that ideally processes cells with high purity, yield and viability. We want to improve the strategy for jet-in-air cell sorting to get more effective cells, and ensure the downstream experiments.

Method: In this study, to achieve high-purity, high-yield, high-viability cell sorting, we explored three aspects. First, we manually adjusted the drop delay through the beads experiment to make it more accurate and to ensure the highest yield in theory, thereby avoiding the influence of the instrument and operator. Second, the effects on cell apoptosis caused by adding $2 \%$ fetal bovine serum to the loading buffer were examined. Third, the effects on cell count, apoptosis, proliferation and gene expression caused by employing FBS and bovine serum albumin in the collecting buffer were analyzed. Statistical analysis was performed with GraphPad Prism 6 software. Significance was set at $p<0.05$.

Result: Our data showed that an additional manual correction of drop delay could lead to an improved cell yield of $98.28 \% \pm 3.79 \%$. Adding FBS $(2 \%)$ to the loading buffer had no significant effect on the fate of sorted cells in 4 hours. However, employing a suitable concentration of FBS/BSA in the collecting buffer mediated a notable increase in cell count from $50 \%$ to $~ 100 \%$, a significant decrease in cell apoptosis from $\sim 40 \%$ to less than $10 \%$ for cell lines and $\sim 70 \%$ to less than $40 \%$ for CD $45+$ primary cells from mouse spleen. And a clear increase in cell proliferation at $48 \mathrm{~h}$ after sorting for jurkat cells. Moreover, the level of gene expression remained steady in the $5 \%$ FBS collecting buffer.

Conclusions: Our manuscript demonstrates techniques that can be easily followed to refine sort yields of healthy cells. Fluorescent-activated cell sorting users could easily obtain enough target cells with great viability and need not to worry about any problems related to collecting or loading buffer. Furthermore, adding extra expensive FBS in collecting or loading buffer to boost yield is no longer necessary. In all, this will make the downstream experiments smoother.

\section{Background}

Flow cytometric sorting is a vital tool in biological research and clinical diagnostics (1). This method functions not only as a technology for isolating target cells but can also provide abundant information on targeted cells, which can help to guide subsequent experiments (2-4). Cell sorting is indispensable because heterogeneous cell suspensions can be purified into fractions containing single cell types based upon virtually unlimited combinations of user-defined parameters (5). Therefore, the cell sorter is the foundation for numerous downstream studies.

Theoretically, a high-end jet-in-air sorter is a fluorescent-activated cell sorting (FACS) sorter that ideally processes cells with high purity, yield and viability (6). However, high-end jet-in-air sorting is a complex process due to its inherent requirements for high fluidic stability and electronic and timing precision. Therefore, a highly accurate sorting strategy to achieve high yield and good biocompatibility, including maintaining high viability and functionality, is a considerable challenge facing high-end jet-in-air sorters. 
It was determined that effective sorting is highly dependent on appropriate drop delay determination. Incorrect determination of the instrument drop delay affects yield and purity (7). Meanwhile, the effect of the collecting buffer was examined. Marie et al. added bovine serum albumin (BSA) to the collecting buffer and notably improved the success of the culture of phytoplankton strains (8). Maxwell et al. demonstrated that the viability and membrane integrity of spermatozoa could be improved by adding seminal plasma into the collecting buffer (9). Therefore, these results suggest that the more similar the collecting buffer is to the living environment of the sorted cells, the higher the cell viability is. However, there was no clear research about adding BSA or fetal bovine serum (FBS) to the collecting buffer during sorting would effect the cell count, apoptosis, proliferation and gene expression. And which concentration of BSA or FBS in the collecting buffer is suitable have not been determined. Moreover, whether adding BSA or FBS in loading buffer would have protective effect during sorting also have not been elucidated.

In this study, we adjusted the drop delay to increase purity and yield. Meanwhile, we investigated the effects on cell count, apoptosis, proliferation and endogenous gene expression caused by adding different concentrations of FBS or BSA to the loading buffer and the collecting buffer during FACS. Based on the data, we suggested the suitable concentration of FBS or BSA for loading buffer and collecting buffer. This strategy is expected to pave the way for further advances in FACS for signal cells and to facilitate downstream experiments after sorting. The strategy is useful for novice users that want to refine their ability to increase yields of healthy cells.

\section{Results}

\section{Effects of drop delay on yield and purity}

We attempted to collect cells with the highest yield using a jet-in-air sorter. To avoid cell loss due to failure or non-optimal calibration of the instrument, we decided to modify the drop delay value using a manual method on an automatic basis to find the accurate drop delay value. The result was that the sort yield was averagely $88.4 \%$ according to the automatic drop delay value, and the yield reached averagely $99.81 \%$ when the drop delay value was modified by the manual method under the same conditions (Fig 1.A). We further analyzed the correlation between drop delay and yield. We confirmed that the drop delay clearly affected the sort yield. For example, when the drop delay was 36.64 , the yield was $97.56 \pm 1.26 \%$. When the drop delay was slightly increased from 36.64 to 36.84 , the yield decreased sharply to $82.67 \pm 5.81 \%$ (Fig 1.B). In addition, if the time duration of the sorting process was longer, for example, reaching $4 \mathrm{~h}$, we could set the drop delay in an optimal setting model to improve the yield rate by 3-5\% (Fig 1.C).

Then, according to the R-max method provided in a previous report (7), the two-way separation experiment was simulated by CST beads to determine the effect of drop delay on the yield and purity of sorted beads. Under the optimal conditions, the R-max value was $90.8 \pm 8.2 \%$. Similarly, the R-max value significantly decreased when the drop delay was offset. However, the purity remained constant and at a high level of almost $100 \%$ regardless of the drop delay value (Fig 1.D-E).

For the living cell experiment, we confirmed the relationship between drop delay and yield by sorting cells into 1536-well plates (Corning). The effect of drop delay on the purity of Jurkat cells was examined by bulk sorting. The yield rate was $46 \%$ when the manual drop delay value was 32.3 . The yield rate decreased to $20 \%$ when the 
drop delay value was shifted to 32.6. The change in the drop delay value had no effect on the sorted cell purity, which was almost $100 \%$ (Fig 1.F).

In the 96-well plate test, the average yield was $87.50 \% \pm 3.76 \%$ under the manual drop delay conditions, and the yield decreased to approximately $52 \%$ when the drop delay values were offset by 0.05 (Supplementary Fig 1 ). Therefore, we can obtain the highest yield by manually adjusting the drop delay.

\section{Effects of FBS in loading buffer on cell apoptosis}

First, to explore the effects of FBS in loading buffer on cell viability, we suspended cells with PBS either without FBS or with $2 \%$ FBS in loading buffer. Then, the cells were sorted into the collecting buffer, which was RPMI1640 medium with or without $10 \%$ FBS, on the optimal drop delay. When the collecting buffer was RPMI-1640 medium only, the apoptosis percentages were $38.05 \pm 2.19 \%$ in the PBS loading buffer group and $42.85 \pm 3.84 \%$ in the $2 \%$ FBS PBS loading buffer group. When the collection buffer was RPMI- 1640 medium with $10 \%$ FBS, the apoptosis percentages were $5.94 \pm 0.78 \%$ in the PBS loading buffer group and $8.14 \pm 0.18 \%$ in the $2 \%$ FBS PBS loading buffer group (Fig 2. A). These data demonstrated that there was no difference between the loading buffers with or without FBS when the cells were collected with the same collecting buffer (Fig 2. C).

Second, we explored whether the incubation time of cells in loading buffer with or without $2 \%$ FBS will affect the cell viability. CD $45+$ cells were incubated in $2 \%$ FBS PBS or PBS at $4^{\circ} \mathrm{C}$ for $2 \mathrm{~h}$ and $4 \mathrm{~h}$, respectively. After incubation, cells were checked the apoptosis rate after sorting. In $2 \%$ FBS PBS loading buffer, the apoptosis percentage was $44.49 \% \pm 6.36 \%$ at $2 \mathrm{~h}$, and was $52.12 \% \pm 6.26 \%$ at $4 \mathrm{~h}$. In PBS loading buffer, the apoptosis percentage was $39.61 \% \pm 6.44 \%$ at $2 \mathrm{~h}$, and was $53.25 \% \pm 6.45 \%$ at $4 \mathrm{~h}$. These data demonstrated that the apoptosis rate increased with the time of sorting, but there was no difference in apoptosis rate between $2 \%$ FBS PBS and PBS loading buffer at $2 \mathrm{~h}$ and $4 \mathrm{~h} \otimes \mathrm{Fig} .2 . \mathrm{B}, \mathrm{D} \otimes$ However, it was clear that the cell apoptosis percentage was significantly decreased by adding FBS to the collection buffer.

\section{Effects on cell count caused by FBS or BSA in the collecting buffer}

On the optimal drop delay and the same other sorted conditions, we suspended cells with PBS and sorted the same cells into a series of collecting buffers. To evaluate the cell viability after sorting, we tested the cell counts, cell apoptosis and cell proliferation under each condition.

The sorted cells were collected with different collecting buffers followed by direct detection of the cell amounts through flow cytometry. The results showed that the actual values of six kinds of collecting buffers were close to the theoretical value, and the yield rate was close to $100 \%$, including for $5 \% \mathrm{FBS}$ in PBS, $10 \%$ FBS and $50 \%$ FBS in medium, 100\% FBS, 0.4\% BSA and 2\% BSA in PBS. For jurkat cells and 293T cells (Fig 3. A-B), the yield rate was at the range of $92.5 \% \sim 104.8 \%$. The yield rate of $0.04 \%$ BSA in the PBS group was $83.7 \% \pm 11 \%$, and the worst group was PBS and medium, exhibiting values of $57.85 \% \pm 14.2 \%$ and $60.3 \% \pm 10.2 \%$, respectively. For primary cells, $\mathrm{CD} 45^{+}$cells from mouse spleen(Fig 3. C), the yield rate was about $93.5 \% \sim 105.5 \%$, and the worst group was also PBS and medium, exhibiting values of $56.99 \% \pm 6.25 \%$ and $54.29 \% \pm 7.31 \%$. In terms of the deviation between the theoretical cell amounts and the actual cell amounts, the amounts decreased more than $40 \%$ when a collecting buffer without FBS or BSA was employed, while the amounts were almost the same when collecting buffers with FBS at the tested concentration or with $2 \%$ BSA were employed. The 
performance of the cell collection buffer was tested by sorting Jurkat cells, 293T cells and CD45+ cells, and we obtained similar results. The specific data of jurkat cells were showed in the Table 1.

\section{Effects of FBS or BSA in collecting buffer on cell apoptosis}

We next assessed the apoptosis rate after sorting with different collecting buffers. Fig 4 shows the apoptosis rates of Jurkat cells (Fig 4. A, D) and 293T cells (Fig 4. B, E). For Jurkat cells, the apoptosis rates of cells collected in PBS and RPMI-1640 medium were $34.4 \pm 6.28 \%$ and $38.1 \pm 2.19 \%$, respectively. The corresponding apoptosis rates of PBS with 5\% FBS and 10\% FBS were $6.95 \pm 0.4 \%$ and $5.73 \pm 0.6 \%$, respectively. The apoptosis rates of $10 \%$ FBS, 50\% FBS and 100\% FBS in the medium groups were $5.94 \pm 0.78 \%, 6.42 \pm 1.3 \%$ and $6.14 \pm 0.13 \%$, respectively. The apoptosis rates of $0.04 \%, 0.4 \%$ and $2 \%$ BSA in the PBS groups were $9.61 \pm 0.5 \%$, $10 \pm 0.6 \%$ and $8.7 \pm 0.6 \%$, respectively. The data of Jurkat cells showed that the collecting buffer with FBS or BSA can maintain high cell viability with apoptosis less than $10 \%$, while the collecting buffer without FBS or BSA increased apoptosis by almost $40 \%$, regardless of medium or PBS. The apoptosis performance of the collecting buffer was also tested by sorting 293T cells and hESC-MSCs (Supplementary Fig 2). We obtained similar results. It should be noted that the apoptosis rate of 293T cells was lower than that of the other tested cells. The apoptosis rate of PBS was $17.59 \pm 1.7 \%$, and the apoptosis rate of medium was $12.6 \pm 1.8 \%$. The apoptosis rate in the $0.04 \%$ BSA group was $6.13 \pm 0.06 \%$. However, the apoptosis rate in the other groups was less than $5 \%$. These results indicated that FBS or BSA in collecting buffers had protective effects on the apoptosis of sorted cells.

For CD $45^{+}$primary cells, the apoptosis rates in PBS and RPMI-1640 medium were $79.25 \% \pm 3.33 \%$ and $73.88 \%$ $\pm 2.37 \%$, respectively. While the apoptosis rates in 5\% FBS and $10 \%$ FBS PBS groups were $39.43 \% \pm 2.50 \%$ and $44.33 \% \pm 8.78 \%$, respectively. Moreover, the apoptosis rates in 10\% FBS, 50\% FBS and 100\% FBS RPMI-1640 medium groups were $40.58 \% \pm 3.19 \%, 36.2 \% \pm 1.77 \%$ and $38.52 \% \pm 4.04 \%$, respectively. The apoptosis rates in $0.04 \%, 0.4 \%$ and $2 \%$ BSA PBS groups were $39.27 \% \pm 5.99 \%, 39.03 \% \pm 4.97 \%$ and $40.6 \% \pm 5.32 \%$, respectively

(Fig 4. C, F). The data showed that the collecting buffer with FBS or BSA can maintain primary cell viability with lower apoptosis rate (average 40\%) also. The apoptosis rate of primary cells after sorting was significantly higher than that of cell lines.

\section{Effects of FBS in collecting buffer on cell proliferation}

We further cultured the sorted Jurkat cells for $48 \mathrm{~h}$, and the growth curve showed that the cells collected by RPMI-1640 with FBS had strong proliferation regardless of the FBS concentration that was used (Fig5).

\section{Collecting buffer with the presence of $5 \%$ FBS can stabilize the genome expression of sorted cells}

To determine the effects of FBS in the collecting buffer on gene expression, we sorted jurkat cells into collecting buffers consisting of PBS either without FBS or with 5\% FBS. We harvested RNA from the cells before and after sorting. The results showed that the gene expression of sorted cells collected with $5 \%$ FBS in the collecting buffer was closer to that of unsorted cells (Fig 6.A). Compared to unsorted cells, cells collected using PBS alone showed 175 up-regulated genes and 35 down-regulated genes. In the presence of 5\% FBS in PBS, the number of affected genes was sharply decreased. There were only 29 up-regulated genes and 8 down-regulated genes. The Venn diagram shows the correlation of affected genes among each group (Fig6.B). The GO 
annotations of affected genes showed that these genes were involved in a wide range of cell functions in biological processes, cellular components and molecular functions (Fig 6.C-E). The top five up- and downregulated genes after sorting using different collecting buffers are listed in supplementary Table 1 and Table 2 . The data demonstrated that the inclusion of $5 \%$ FBS in the collecting buffer significantly protected the inherent gene expression of jurkat cells during sorting.

\section{Discussion}

An accurate drop delay assessment is critical to achieve successful cell sorting with high purity and yield (12). Our results demonstrate that the yield of sorting was improved by manually adjusting the drop delay. This approach was also used in the analysis of beads and cells, and the results were better than those of previous reports (13). The results demonstrated that a precise drop delay can obtain the highest sorting yield and purity. Deviation of drop delay in a narrow region can maintain perfect purity but sharply decrease yield. In other words, yield is considerably more sensitive to the deviation of drop delay than purity is. However, in an experiment on long-term sorting, the drop delay may be shifted slightly due to the status of the sorter changing, even if the sorter can still maintain sorting. This finding may be attributable to the decrease in sorting yield of approximately $3 \%$ to $5 \%$ (Fig 1.C). In this case, the way to improve sorting yield is to recalculate the drop delay. In this study, we suggest that if a single cell needs to be sorted after continuous sorting for 3-4 $\mathrm{h}$, it is better to recalculate the drop delay to obtain a better yield. For most FACS sorting, especially for the sorting of single cells, rare populations or precious samples, the primary goal is to obtain as many cells of interest as possible based on good purity. Therefore, guaranteeing a precise drop delay during sorting is indispensable for obtaining a high sorting yield. In addition, experienced instrument operators can obtain similar to manual mode drop delay through automatic mode, similar to the result in the study, but for beginners, because of the uncertainty increase, manual adjustment is more essential.

The R-max method holds that evaluating the actual recovery of a given sort typically relies on direct measurements of the absolute number of target particles in the sorted and original fractions. To validate whether the manual method makes the equipment with the highest yield, R-max values on the manually modified drop delay were compared with those that were reported in the article. The data showed that the Rmax value with the manually modified drop delay was higher. By manually adjusting the drop delay described in this paper, the instrument can achieve the highest yield.

The decrease in cell viability during sorting may primarily due to the pressure and strong electric field associated with the sorting process $(14,15)$. Our results showed that $2 \%$ FBS in the loading buffer had almost no effect on yield and purity in 4 hours. We hypothesized that this lack of effect might be observed because the cells are not damaged by the sorting process; in fact, the cells were healthy. Therefore, utilizing loading buffer with or without FBS might have little influence on cell conditions. However, importantly, we did observe that including FBS in the collecting buffer does have a strong influence on the viability of cells, rather than that in the loading buffer. The addition of $10 \%$ FBS or $2 \%$ BSA in the collecting buffer considerably increased the cell amounts, decreased apoptosis and promoted the proliferation of sorted cells (Table 1).

In recent years, many important cellular characteristics have been assessed specifically with the help of singlecell approaches. High-throughput single-cell transcriptomics has provided unprecedented insights into the 
cellular diversity of tissue. For this method, maintaining gene expression is critical. Graham M. Richardson used a microarray to show that the selection of the sorter instruments was considerably less important than other factors related to how cells are isolated and handled with regard to short-term gene expression (16). However, our data showed that including FBS in the collection buffer had an effect on gene expression. Paired analysis of gene expression showed significant differences between the two groups. These results suggest that adding 5\% FBS to the collecting buffer is as important as maintaining a temperature of $4^{\circ} \mathrm{C}$ to minimize the gene expression changes through the duration of the sorting process $(16,17)$.

"garbage in, garbage out" is suitable for all sorting experiments, so the state of the cells before sorting plays a decisive role in addition to the factors mentioned in the article that affect the viability. For 96 -plate single-cell sorting, the position of the plate is also a key factor to be considered.

\section{Conclusions}

Our results strongly suggest that the concentration of FBS or BSA in the collection buffer was crucial for downstream experiments, including cell culture and genomic expression experiments. We strongly recommend adding 5\% FBS, 10\% FBS or 2\% BSA in the collecting buffer, either in PBS or in culture medium, depending on the experimental design (Fig 7).

In summary, our study demonstrates that an effective strategy can be employed to perform sorting with high yield, viability and gene expression stability. Furthermore, researchers may adjust the collecting buffer for sorting based on their downstream experiments and the results of these modifications to cell sorting parameters, which have exhibited notable improvements with the minor adjustments that we made.

\section{Methods}

\section{Beads}

Calibration beads for MoFlo Astrios (Beckman Coulter, Inc. Miami USA) were diluted according to the manufacturer's instructions during the cell sorter setup procedures to maximize the instrument's optical alignment. Flow-Check ${ }^{\mathrm{TM}}$ beads (Beckman Coulter, Inc., Miami, USA) were used directly without dilution during the identification of the instrument drop delays. CS\&T research beads (BD Biosciences San Jose CA USA) were used directly without dilution during the identification of the instrument drop delay by the R-max method.

\section{Cell Culture}

Jurkat cells were cultured in RPMI-1640 medium and Human ESC-MSCs and 293T cells were cultured in Dulbecco's modified Eagle's medium (DMEM) $₫$ supplemented with 10\% fetal bovine serum (FBS) and $1 \%$ penicillin and streptomycin in a $5 \%(\mathrm{v} / \mathrm{v}) \mathrm{CO}_{2}$ incubator at $37^{\circ} \mathrm{C}$.

\section{Isolation of $\mathrm{CD}_{4} 5^{+}$cells from spleen}

To isolate $\mathrm{CD} 45^{+}$cells, four 8-week male C57BL/6 mice were sacrificed. All spleens were dissected and collected into digestive solution containing $0.5 \mathrm{mg} / \mathrm{ml}$ papain (Worthington, Cat: LS003119) and $0.5 \mathrm{mg} / \mathrm{ml}$ collagenase type 2 (Worthington, Cat: LS004176) in high glucose DMEM medium for 10 min. Cells were passed through a 
$40 \mathrm{~mm}$ cell strainer (FALCON, ref352340) to create single cell suspension. Cells were spun down (500 g) at $4^{\circ} \mathrm{C}$ for $5 \mathrm{~min}$, the supernatant was discarded. Cells were suspended in $3 \mathrm{ml}$ red blood cell lysis buffer (BioLegend, Cat: 420301) for $3 \mathrm{~min}$, then $6 \mathrm{ml}$ PBS were added to stop lysing. Next, cells were spun down $(500 \mathrm{~g})$ at $4^{\circ} \mathrm{C}$ for $5 \mathrm{~min}$. After the supernatant was discarded, cells were suspended in $200 \mathrm{ml}$ phosphate buffered saline (PBS) for $\mathrm{CD} 45^{+}$staining. To stain the cells for sorting, the cells were incubated with anti-mouse $\mathrm{CD} 45^{+}$monoclonal antibody conjugated with PE/Cyanine7 fluorophores (BioLegend, Cat:103114) at $4^{\circ} \mathrm{C}$ for 45 min. After wash, cells were resuspended in PBS to $10^{7}$ cells / $\mathrm{ml}$ for next step experiment.

\section{CFSE staining and Hoechst 33342 staining}

Prior to sorting, cells were suspended in PBS at a concentration of $1 \times 10^{6}$ cells $/ \mathrm{ml}$. Cells were labeled with carboxyfluorescein diacetate (CFSE, C34554, Invitrogen, final concentration $5 \mu \mathrm{M}$ ) at a concentration of $2 \times 10^{6}$ cells/ml in RPMI-1640 or DMEM medium with $1 \mu$ l CFSE for 10 min at room temperature, and the reaction was subsequently stopped by washing three times using RPMI-1640 or DMEM medium containing 10\% FBS; cells were later suspended in $1 \mathrm{ml}$ PBS, and $1 \mu$ l Hoechst 33342 (Sigma) was added to the suspension. A mixture of stained cells and unstained cells with a volume ratio of 1:1 was diluted in PBS buffer (cell concentration: $10^{5}-$ $10^{6} \mathrm{cells} / \mathrm{ml}$ ) before sorting.

\section{Flow cytometry for cell sorting}

A MoFlo Astrios EQ flow cytometer (Beckman Coulter, Inc. Miami USA) is a high-end jet-in-air sorter equipped with a $488 \mathrm{~nm}$ laser set to $200 \mathrm{~mW}$ at the laser intersection point, and a standard filter setup was used for cell sorting. In this study, the instrument was configured with a 100- $\mu \mathrm{m}$ nozzle at $30 \mathrm{psi}(210 \mathrm{kPa})$ and $49 \mathrm{kHz}$ droplet generation. The sample offset was set at $0.3 \mathrm{psi}$ and was kept constant for the duration of the experiment. Sorting was performed using a 1-drop and 0.5-drop deflection sort mode to give the minimal condition of coincidence events (7). During the sorting process, the sample and collection tubes were kept at $5^{\circ} \mathrm{C}$. Beckman Coulter Summit software was used to control the instrument.

\section{Flow cytometry for cell count and apoptosis analysis}

The flow cytometry instruments included Cytoflex LX (Beckman Coulter, Inc., Miami, USA), LSRFortessa (BD Biosciences) and Novocyte (ACEA Novocyte3000) equipped with a sampler, which were used to count cells and to examine cells for apoptosis after sorting.

\section{Setting of the drop delay}

There are two methods used to set the drop delay for the MoFlo Astrios EQ sorter. Based on the stability of the fluidics, the automatic drop delay determination was established using an Intellisort with automatic drop delay determination. The manual method modified the automatic drop delay in the manner described in the manufacturer's instructions (8-9,11-12). The drop delay was calculated by sorting beads on slides and viewed by microscopy. Flow check TM beads were run at 100 events per second (eps) to ensure approximately $100 \%$ sort efficiency. The laser was set to $200 \mathrm{~mW}$ of power. The sort decision was made on a Forward Scatter (FSC) vs Side Scatter (SSC) population. The drop delay by manual method was identified as the one giving the most beads in the $5^{\text {th }}$ puddle and nothing in both the $4^{\text {th }}$ and $6^{\text {th }}$ puddles. 


\section{Yield assay by fluorescence for beads}

The yield according to the setting of drop delay was further investigated. Using automatic drop delay and manually adjusted drop delay separately, ten different drop delay settings in series were tested with 0.1 intervals. The trend of yield around the optimal drop delay $( \pm 0.05)$ was obtained with 0.01 intervals. All experiments were performed under Drop Delay Wizard mode, and each drop delay setting was used to sort 50 droplets on glass slides. The number of fluorescent beads in each puddle on each glass slide was counted using a microscope.

\section{Yield and purity assay by R-max method for CST beads}

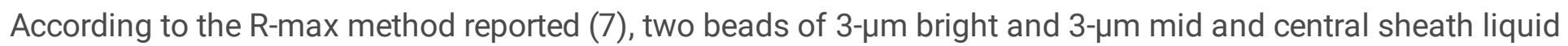
flow were sorted at the same time. After the three kinds of solutions were detected by flow cytometry, the R-max value and the purity of the two kinds of beads were calculated.

\section{Yield and purity assay for single cells}

For the living cell experiment, we assayed the yield by sorting one cell into each well of 1536-well plates. Jurkat cells were stained by CFSE and Hoechst 33342. A mixture of stained cells and unstained cells with a volume ratio of 1:1 was diluted in PBS buffer (cell concentration: $10^{5}-10^{6}$ cells per $\mathrm{ml}$ ). White 1536 -well plates (Corning) were prepared by adding $5 \mu$ of PBS to each well. After sorting, the plate was removed and centrifuged to keep the cell on the bottom of the well. Each well of a 1536-well plate was scanned with a BioTek Cytation 1 Cell Imaging Multi-Mode Reader individually.

The purity assay was performed as follows. CFSE-stained Jurkat cells were mixed at 1:1 with unstained cells to obtain a cell suspension $\left(1 \times 10^{7} \mathrm{cell} / \mathrm{ml}\right)$. Changing the drop delay value, 30,000 negative and positive cells were collected using each different drop delay value. Then, the cell purity was detected by flow cytometry.

\section{Sorting buffer}

Most experiments used 2\% FBS in PBS as the loading buffer. Two kinds of loading buffers were tested in this study: PBS and $2 \%$ FBS in PBS.

FBS is generally added to the collecting buffer. Because the content of FBS is complex, some experiments are not applicable. In this case, those experiments use BSA instead of FBS. There were three groups in this work: (i) FBS in cell culture medium, with FBS concentrations of $0 \%, 10 \%, 50 \%$ and $100 \%$ being employed. We used Jurkat, 293T cells and CD45+ cells from mouse spleen; therefore, the culture medium included RPMI-1640 and DMEM. (ii) FBS in PBS, with FBS concentrations of $0 \%, 5 \%$ and $10 \%$ being employed. (iii) BSA in PBS, with BSA concentrations of $0 \%, 0.04 \%, 0.4 \%$ and $2 \%$ being employed.

\section{Cell counts assay}

We investigated the effect of the collecting buffer on the actual cell amounts. We tested three kinds of cells: Jurkat cells, 293T cells and CD45+ cells from mouse spleen. We sorted 500,000 cells into different groups of collecting buffer. Fifty microliters of collecting buffer containing sorted cells was directly detected using flow cytometry to count the actual cell amounts. The theoretical cell amounts in $50 \mu l$ collecting buffer were 
calculated based on the cell amounts of 500,000 and the weight difference between the collection tube after sorting and the empty tube. The density of each collecting buffer was considered to be $1 \mathrm{~g} / \mathrm{ml}$. The yield rate is defined as "the actual cell amounts/the theoretical cell amounts".

\section{Apoptosis assay}

The cells were collected by the corresponding collecting buffer. Cell apoptosis was detected immediately after

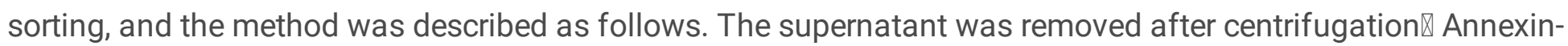
V-FITC/PI staining was performed as previously described (11). Two-dimensional flow cytometry was performed to detect early and late apoptotic cells. The percentage of cell apoptosis represented both early and late apoptotic cells.

\section{Cell proliferation assay by CCK-8}

Cell proliferation was examined by a Cell Counting Kit-8 (CCK-8, APExBIO Company). After the cells were collected with different collecting buffers, 2000 cells were seeded in each well of a 96 -well plate. We measured the OD450 value by an M5 microplate reader at 24 and $48 \mathrm{~h}$.

\section{Cell proliferation assay by single-cell sorting of $96-$ well plates}

Before sorting, 96-well plates (Corning) were prepared by adding $200 \mu \mathrm{l}$ of culture medium to each well. Jurkat cells were suspended in PBS. In this test, one cell was sorted into one well of the plate using three drop delay values. The three drop delay values included the optimal drop delay obtained by manual adjustment, and the other two drop delay values were obtained by adding and subtracting 0.05 from the optimal value. We tested all wells in the plate using each drop delay value and repeated one plate. After sorting, we placed the plate in a $37^{\circ} \mathrm{C}$ incubator with $5 \% \mathrm{CO}_{2}$ for one week. We counted the numbers of clones using microscopy.

\section{Gene sequencing}

For some experiments, it is necessary to purify cells for gene sequencing by FACS; therefore, we investigated whether adding FBS to the collecting buffer affects the gene expression characteristics of sorted cells. We established two groups: the PBS group and the 5\% FBS in PBS group. Jurkat cells were sorted into the PBS and $5 \%$ FBS in PBS buffers. Then, $1 \times 10^{6}$ cells were sorted in 5 min and collected to extract total RNA. A library was built after enrichment purification using magnetic beads with Oligo (dT), and a quality library was sequenced via the Illumina platform with a pe150 sequencing strategy. The difference expression multiple was 2 . The Pvalue was 0.05 , and padj was 0.05 . To detect the difference in gene function caused by sorting, GO and KEGG were used to analyze the sequencing results.

\section{Statistical analysis}

Data are presented as the mean \pm SEM and are representative of 3 independent experiments. Statistical analysis was performed with GraphPad Prism 6 software. One-way analysis of variance (ANOVA) with the Newman-Keuls post hoc test or two-way repeated measures ANOVA with the Bonferroni post hoc test were used for comparison of multiple groups. Significance was set at $p<0.05$. 


\section{List Of Abbreviations}

\begin{tabular}{|ll|}
\hline FACS & fluorescent-activated cell sorting \\
\hline FBS & fetal bovine serum \\
\hline PBS & phosphate buffered saline \\
BSA & bovine serum albumin \\
\hline CSC & central sheath catch \\
\hline DMEM & Dulbecco's modified Eagle's medium \\
\hline CFSE & carboxyfluorescein diacetate \\
\hline FSC & Forward Scatter \\
\hline SSC & Side Scatter \\
\hline
\end{tabular}

\section{Declarations}

\section{Ethics approval and consent to participate:}

The experimental procedures were approved by the Animal Ethics Committee of Zhejiang University and were carried out in accordance with National Institutes of Health guidelines.

\section{Consent for publication:}

Not applicable.

\section{Availability of data and material:}

All data generated or analysed during this study are included in this published article [and its supplementary information files].

The raw data of flow cytometry generated during the current study are available in the [flowrepository] repository, [http://flowrepository.org/id/RvFrOFmdMexjxQEThko3SmNcpZYicu30v5aoGEYYu6E61oTszrBqSc5i9jm4FYA4], and the gene sequence had been submitted on NCBI, and the number is SUB8446820.

\section{Competing interests:}

All of authors declare that we have no competing interests.

\section{Funding:}

The project of Zhejiang Science and Technology Department (No. LGF19H150006). The project of Experimental Technology of Zhejiang University laboratory department (No. SJS201712) and the project of Zhejiang Provincial Department of Education (No. Y201941524, No. 201942279). This study was supported by the National Natural Science Foundation of China (No. 81972138, No. 81572229, No.81501943). 
Authors' contributions:

\begin{tabular}{ll} 
Authors' Name & contributions \\
\hline Linlin Wang & design of the work, drafted the work or substantively revised it \\
\hline Xinghui Song & design of the work, the acquisition, analysis, and interpretation of data \\
\hline Jiajia Wang & the analysis and interpretation of data \\
\hline Yanwei Li & the acquisition of data \\
\hline Yueting Xing & the acquisition of data \\
\hline Chun Guo & the acquisition of data \\
\hline Yingying Huang & the acquisition of data \\
\hline Lintao Xu & the acquisition of mouse cells \\
\hline Hu Hu & drafted the work or substantively revised it \\
\hline
\end{tabular}

\section{Acknowledgements:}

Thanks to the core facilities of Zhejiang University school of medicine for providing instruments and equipment for research.

\section{Authors' information:}

${ }^{*}$ Corresponding Author: Lin-lin Wang, PhD, MD

Department of Basic Medicine Sciences, and Department of Orthopaedics of Sir Run Run Shaw Hospital, Zhejiang University School of Medicine, Hangzhou, Zhejiang 310058, China

Tel and Fax: 0086-571-88208250

E-mail: wanglinlin@zju.edu.cn

${ }^{*}$ Co-corresponding Author: $\mathrm{Hu} \mathrm{Hu,} \mathrm{PhD,} \mathrm{MD}$

Department of Pathology and Pathophysiology, Zhejiang University School of Medicine, Hangzhou, Zhejiang 310058, China

Tel and Fax: 0086- 0571-88208715

E-mail addresses: huhu@zju.edu.cn

The first Author: Xinghui Song

Core facilities, Zhejiang University School of Medicine, Hangzhou, Zhejiang 310058, China

Tel: 0086-0571-88981622 
The other Authors: Jiajia Wang, Yanwei Li, Yueting Xing, Chun Guo, Yingying Huang

Core facilities, Zhejiang University School of Medicine, Hangzhou, Zhejiang 310058, China

Tel: 0086-0571-88981951

The Author: Lintao Xu

Department of Neurosurgery, Second Affiliated Hosptical of Zhejiang University School of Medicine, Hangzhou, China

Tel: 0086-0571-87784714

\section{References}

1. Davis MP. PC-FACS. J Pain Symptom Manage. 2018;55(1):172-8.

2. Geslewitz WE, Percopo CM, Rosenberg HF. FACS isolation of live mouse eosinophils at high purity via a protocol that does not target Siglec F. Journal of Immunological Methods. 2018;454:27-31.

3. Chen P, Feng X, Du W, Liu BF. Microfluidic chips for cell sorting. Front Biosci. 2008;13:2464-83.

4. Swierczak A, Pollard JW. FACS isolation and analysis of human circulating and tumor neutrophils. Methods Enzymol. 2020;632:229-57.

5. Zhang X, Feng J, Wang H, Zhu J, Zhong Y, Liu L, et al. Bivariate flow cytometric analysis and sorting of different types of maize starch grains. Cytometry A. 2018;93(2):213-21.

6. Omana-Zapata I, Mutschmann C, Schmitz J, Gibson S, Judge K, Aruda Indig M, et al. Accurate and reproducible enumeration of T-, B-, and NK lymphocytes using the BD FACSLyric 10-color system: A multisite clinical evaluation. PLoS One. 2019;14(1):e0211207.

7. Riddell A, Gardner R, Perez-Gonzalez A, Lopes T, Martinez L. Rmax: A systematic approach to evaluate instrument sort performance using center stream catch. Methods. 2015;82:64-73.

8. Marie D, Le Gall F, Edern R, Gourvil P, Vaulot D. Improvement of phytoplankton culture isolation using single cell sorting by flow cytometry. J Phycol. 2017;53(2):271-82.

9. W. M. C. Maxwell GRWaLAJ. <Viability and Membrane Integrity of Spermatozoa After Dilution and Flow Cytometric Sorting in the Presence or Absence of Seminal Plasma.pdf>. Reprod Fertil Dev. 1997;8:1165-78.

10. culter b. <MoFlo Astrios EQ IFU B22986B new.pdf>. 2013.

11. Dankert JF, Rona G, Clijsters L, Geter P, Skaar JR, Bermudez-Hernandez K, et al. Cyclin F-Mediated Degradation of SLBP Limits H2A.X Accumulation and Apoptosis upon Genotoxic Stress in G2. Mol Cell. 2016;64(3):507-19.

12. Lazebnik YA, Poletaev Al, Zenin VV. Drop-delay measurement using enzyme-coated particles. Cytometry. 1992;13(6):649-52.

13. Osborne GW. A method of quantifying cell sorting yield in "real time". Cytometry A. 2010;77(10):983-9.

14. Osborne GW. Recent advances in flow cytometric cell sorting. Methods Cell Biol. 2011;102:533-56.

15. Pritchard RH, Zhukov AA, Fullerton JN, Want AJ, Hussain F, la Cour MF, et al. Cell sorting actuated by a microfluidic inertial vortex. Lab Chip. 2019;19(14):2456-65. 
16. Richardson GM, Lannigan J, Macara IG. Does FACS perturb gene expression? Cytometry A. 2015;87(2):166-75.

17. Wylot B, Konarzewska K, Bugajski L, Piwocka K, Zawadzka M. Isolation of vascular endothelial cells from intact and injured murine brain cortex-technical issues and pitfalls in FACS analysis of the nervous tissue. Cytometry A. 2015;87(10):908-20.

\section{Tables}

Table 1: Effects of the additions of FBS/BSA in collecting buffer on cell counts and apoptosis for jurkat cell.

\begin{tabular}{|c|c|c|c|c|c|c|c|c|c|}
\hline \multirow{2}{*}{ collecting buffer } & \multicolumn{2}{|c|}{ FBS in PBS } & \multicolumn{3}{|c|}{ BSA in PBS } & \multicolumn{4}{|c|}{ FBS in RPIM-1640 } \\
\hline & $\mathbf{0 \%}$ & $\mathbf{5 \%}$ & $0.04 \%$ & $0.40 \%$ & $2 \%$ & $\mathbf{0 \%}$ & $10 \%$ & $50 \%$ & $100 \%$ \\
\hline $\begin{array}{c}\text { actual } \\
\text { amounts }(50 u l)\end{array}$ & $\underline{6135}$ & 9272 & 7903 & 8971 & 9506 & $\underline{\mathbf{5 7 1 5}}$ & 9733 & 9864 & 10299 \\
\hline $\begin{array}{c}\text { theoretical } \\
\text { amounts(50ul) }\end{array}$ & 9085 & 9330 & 9860 & 9697 & 9609 & 9526 & 9519 & 9556 & 9819 \\
\hline Apoptosis (\%) & \multicolumn{6}{|c|}{ 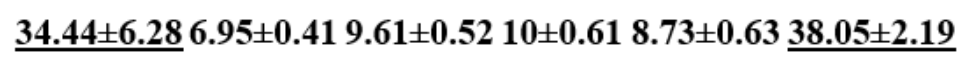 } & $5.94 \pm 0.78$ & $6.42 \pm 1.29$ & $6.14 \pm 0.13$ \\
\hline
\end{tabular}

\section{Figures}



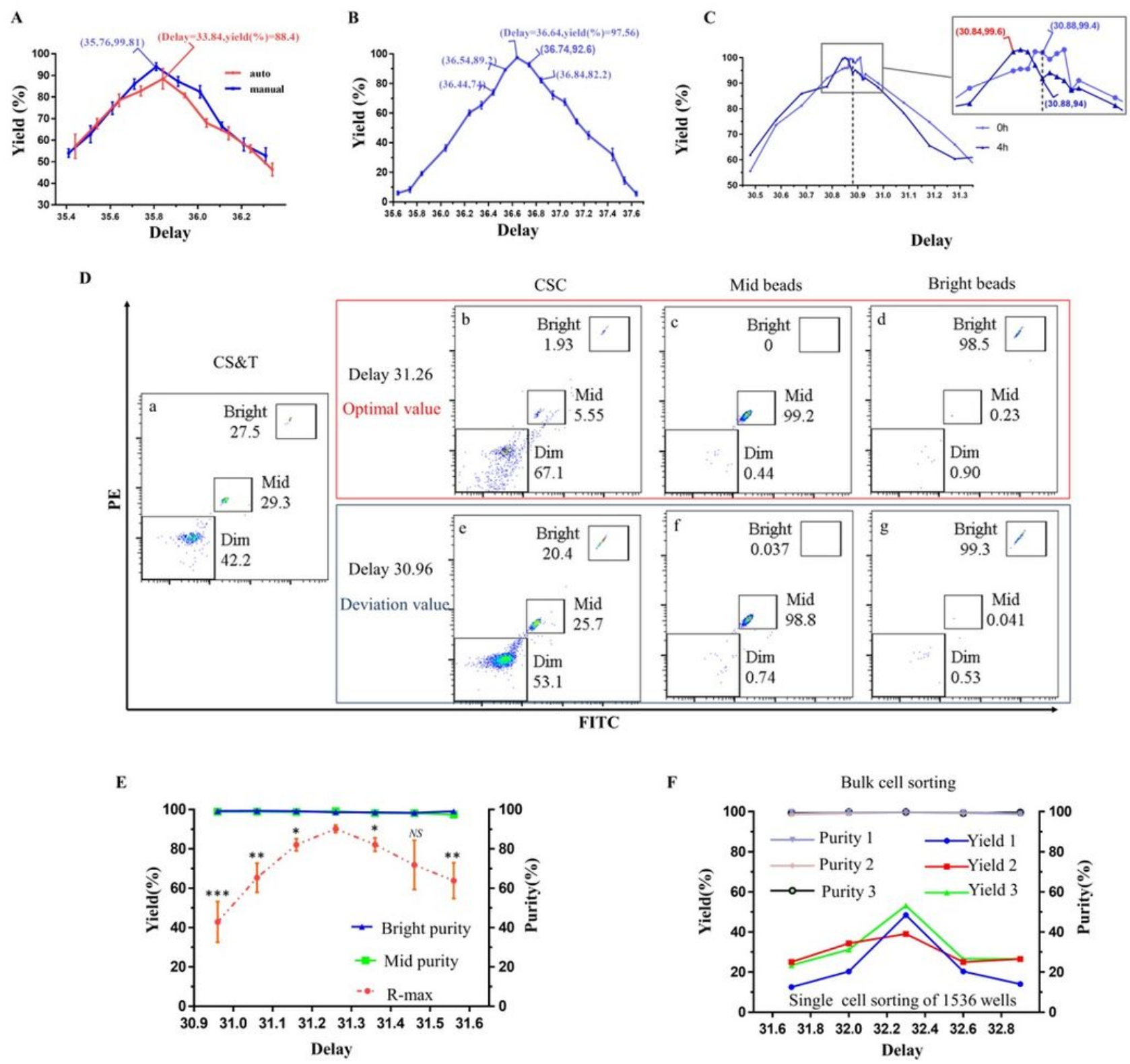

FITC

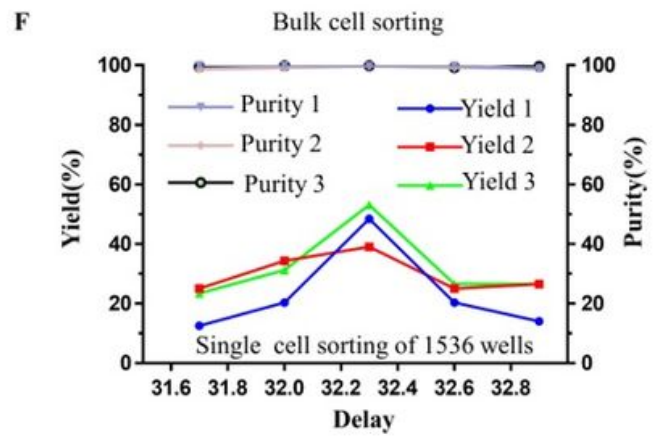

\section{Figure 1}

Effects of drop delay on the yield and purity of sorted cells and beads. The effect of drop delay on the yield of beads was tested. Each of the ten puddles was collected using a drop delay setting with 0.1 intervals. Precisely 50 droplets were deposited into each puddle at different drop delay settings. A. The effect of drop delay on the yield was tested with automatic and manually adjusted drop delays. The red line is the automatic one, and the blue line is the other one. B. Map of the correlation between drop delay and yield. C. Effect of long-term sortingrelated drop delay deviation on yield. D - E. The R-max method was used to assess the effect of drop delay on yield and purity. CS\&T beads consist of 3- $\mu \mathrm{m}$ bright or mid, and $2-\mu \mathrm{m}$ dim beads. According to the R-max method reported, two beads of 3- $\mu$ m bright or mid and central sheath catch (CSC) were sorted at the same time. After flow cytometry detection, the R-max value and purity of two kinds of beads were analyzed. D. Dot plots of flow cytometry detection. a. Dot plots of CS\&T beads. b - g. Dot plots of CSC, mid and bright beads were sorted 
with delay value 31.26 (b-d, red frame) and 30.96 (e-g, blue frame) .31 .26 is the optimal value. 30.96 is the Value of deviation. F. The yield and purity of cells were assessed under manually adjusted conditions. Jurkat cells stained with CFSE and Hoechst 33342 were mixed at 1:1 with unstained cells to obtain a suspension ( $1 \mathrm{x}$ 107 cells $/ \mathrm{ml}$ ). The double-positive cells were sorted into 1536-well plates. Then, each well of cells in the 1536well plate was scanned with a BioTek Cytation 1 Cell Imaging Multi-Mode Reader and imaged individually. The data were analyzed by GEN5 3.04 software. Each experiment was repeated at least 3 times.
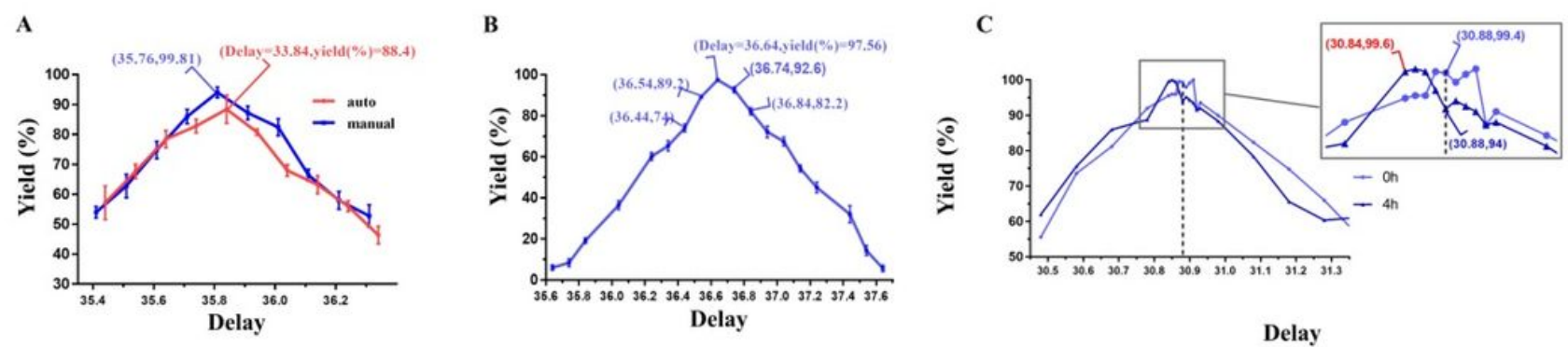

D

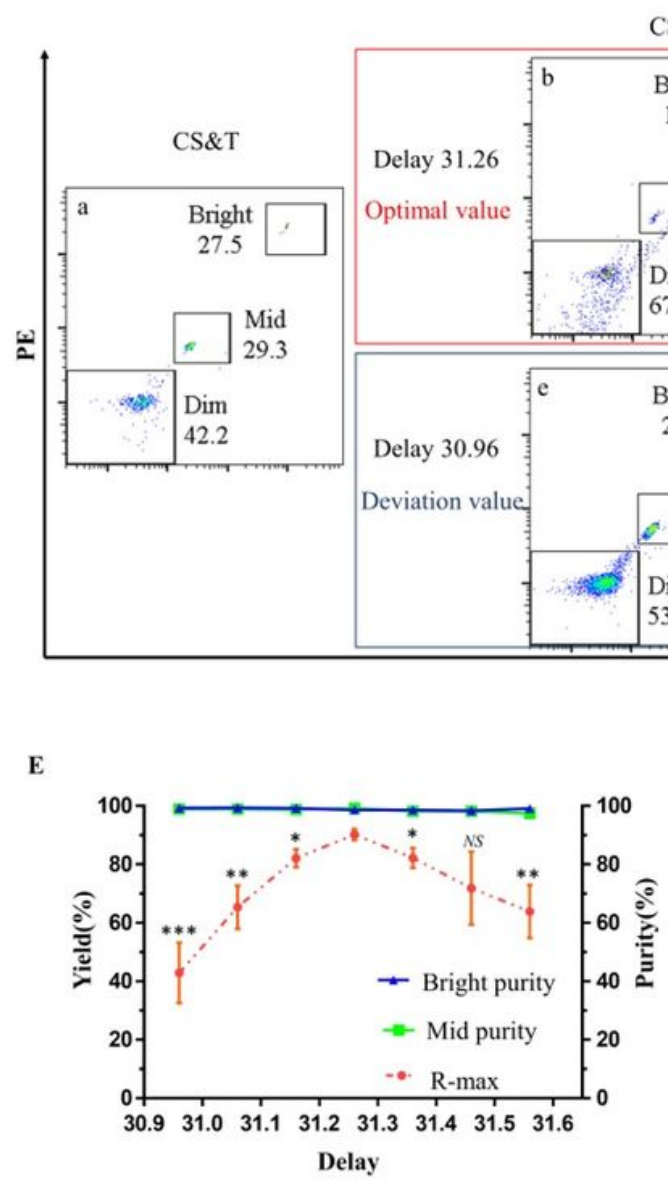

CSC Mid beads Bright beads
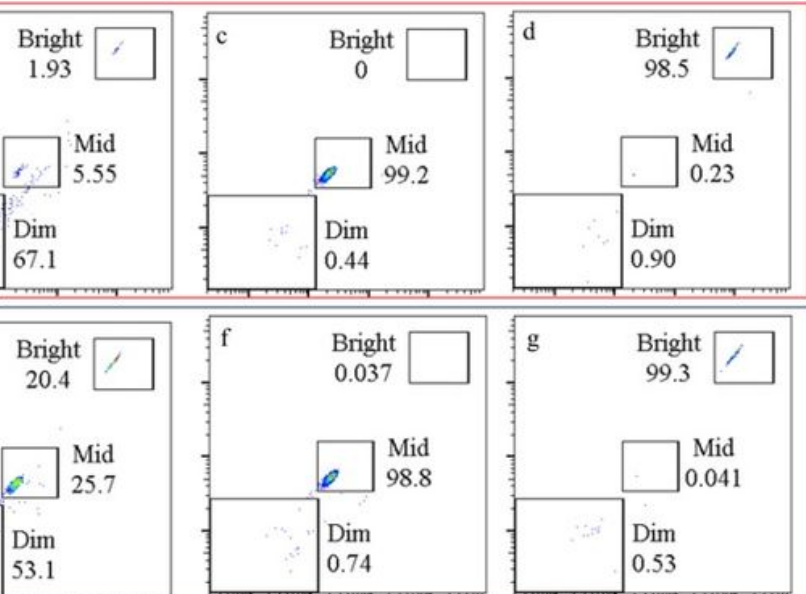

FITC

$\mathbf{F}$

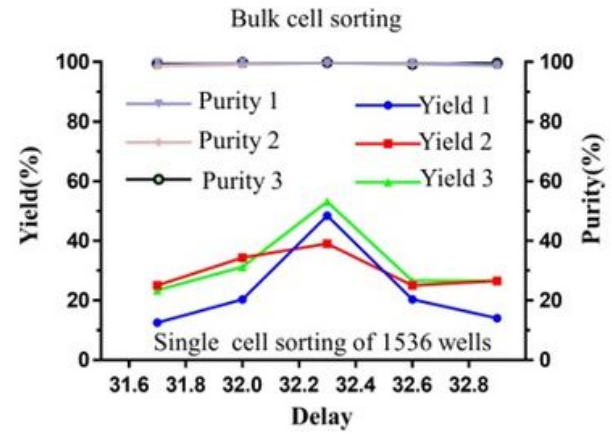

\section{Figure 1}

Effects of drop delay on the yield and purity of sorted cells and beads. The effect of drop delay on the yield of beads was tested. Each of the ten puddles was collected using a drop delay setting with 0.1 intervals. Precisely 50 droplets were deposited into each puddle at different drop delay settings. A. The effect of drop delay on the yield was tested with automatic and manually adjusted drop delays. The red line is the automatic one, and the 
blue line is the other one. B. Map of the correlation between drop delay and yield. C. Effect of long-term sortingrelated drop delay deviation on yield. D - E. The R-max method was used to assess the effect of drop delay on yield and purity. CS\&T beads consist of 3- $\mu \mathrm{m}$ bright or mid, and $2-\mu \mathrm{m}$ dim beads. According to the R-max method reported, two beads of 3- $\mu \mathrm{m}$ bright or mid and central sheath catch (CSC) were sorted at the same time. After flow cytometry detection, the R-max value and purity of two kinds of beads were analyzed. D. Dot plots of flow cytometry detection. a. Dot plots of CS\&T beads. b - g. Dot plots of CSC, mid and bright beads were sorted with delay value 31.26 (b-d, red frame) and 30.96 (e-g, blue frame) 31.26 is the optimal value. 30.96 is the Value of deviation. F. The yield and purity of cells were assessed under manually adjusted conditions. Jurkat cells stained with CFSE and Hoechst 33342 were mixed at 1:1 with unstained cells to obtain a suspension ( $1 \mathrm{x}$ 107 cells $/ \mathrm{ml}$ ). The double-positive cells were sorted into 1536-well plates. Then, each well of cells in the 1536well plate was scanned with a BioTek Cytation 1 Cell Imaging Multi-Mode Reader and imaged individually. The data were analyzed by GEN5 3.04 software. Each experiment was repeated at least 3 times.

A

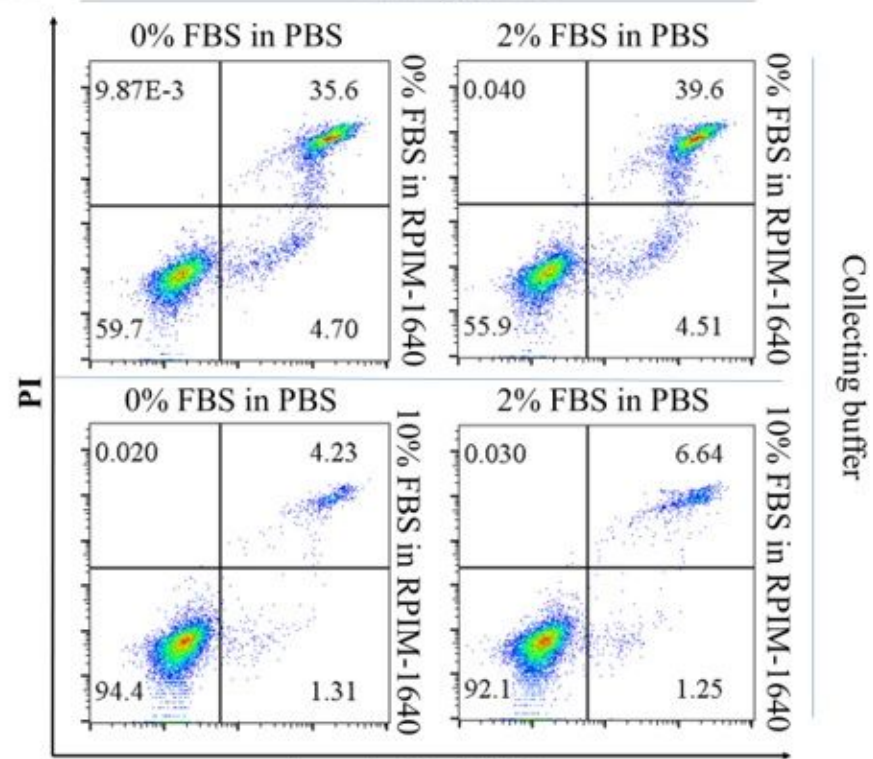

Annexin V - FITC

C

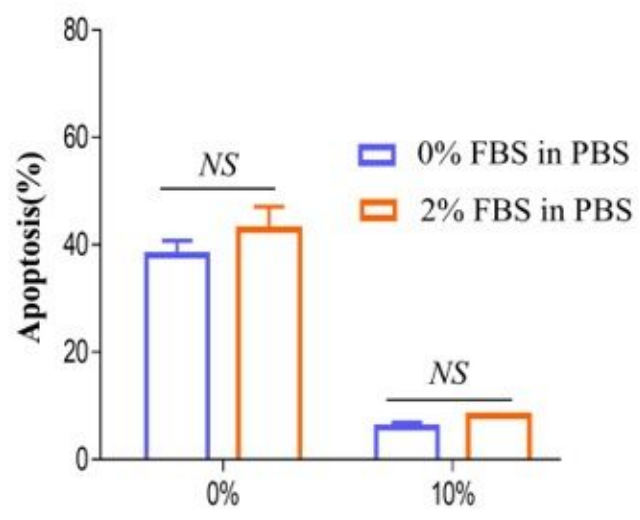

Concentration of FBS in RPMI-1640
B

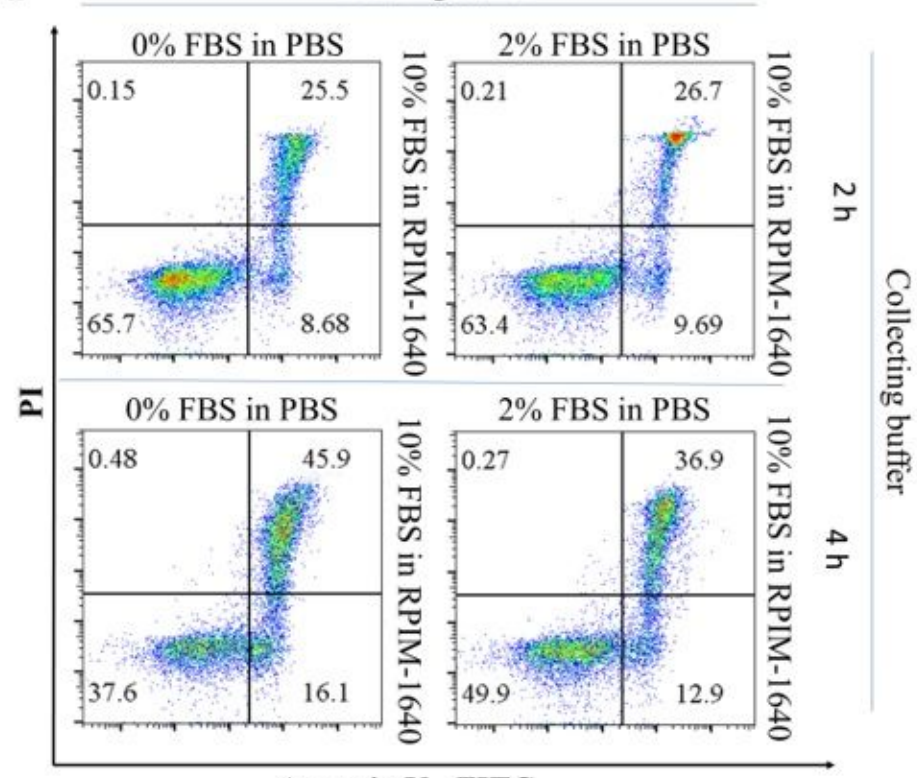

Annexin V - FITC

D

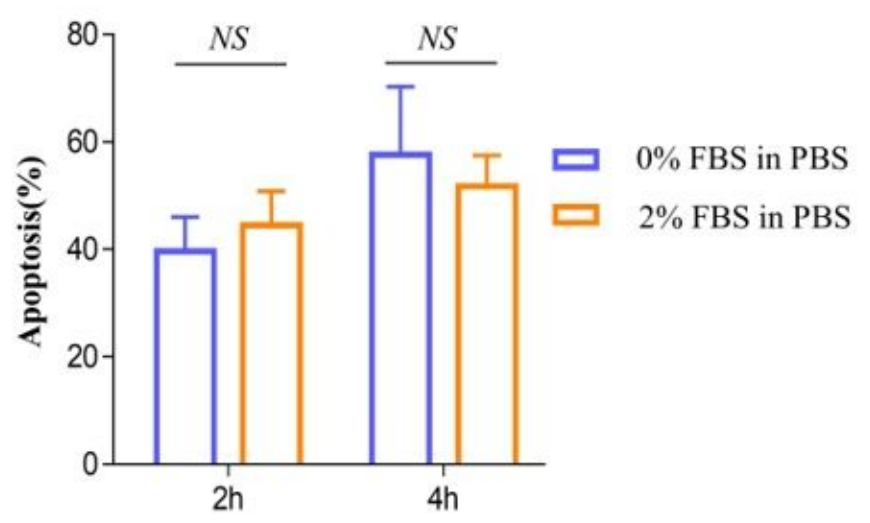

Time of cells in loading buffer

Figure 2

Effects of the addition of FBS in loading buffer on cell apoptosis. A. Dot plots of apoptotic cells sorted with different loading and collecting buffers. B. Dot plots of apoptotic cells sorted with different loading and 
collecting buffers after 2 hours and 4 hours. Numbers in quadrants indicate the percentages of cells in each throughout. Cells were stained with Annexin- $V$ and propidium iodide and analyzed by flow cytometry. C-D. The data of the percentage of apoptotic cells of three independent experiments. Data are presented as the mean \pm SEM. Each experiment was repeated at least 3 times.

A

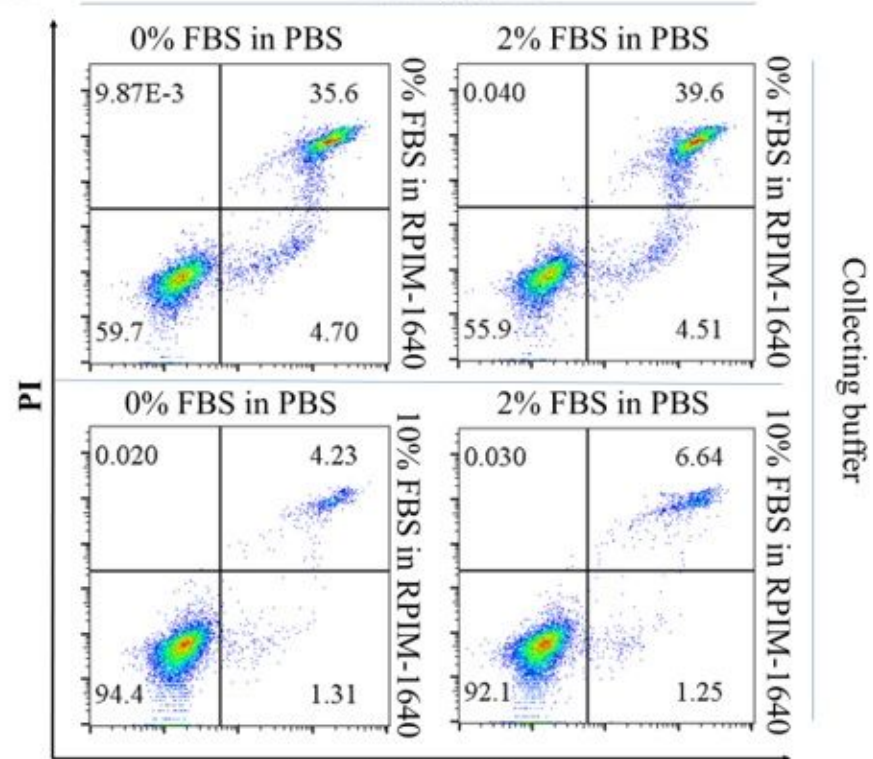

Annexin V - FITC

C

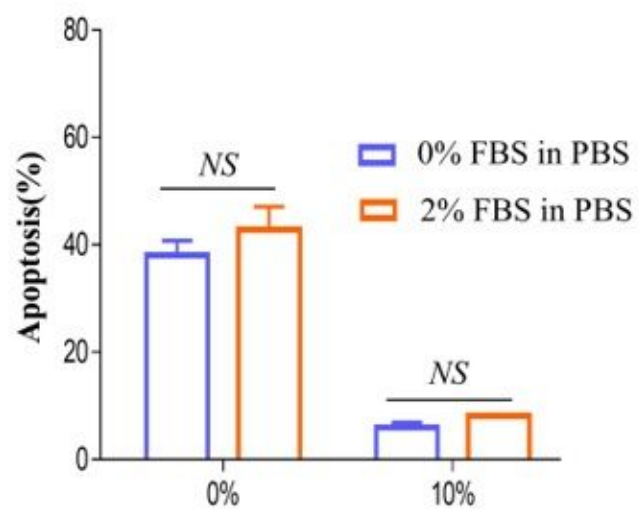

Concentration of FBS in RPMI-1640

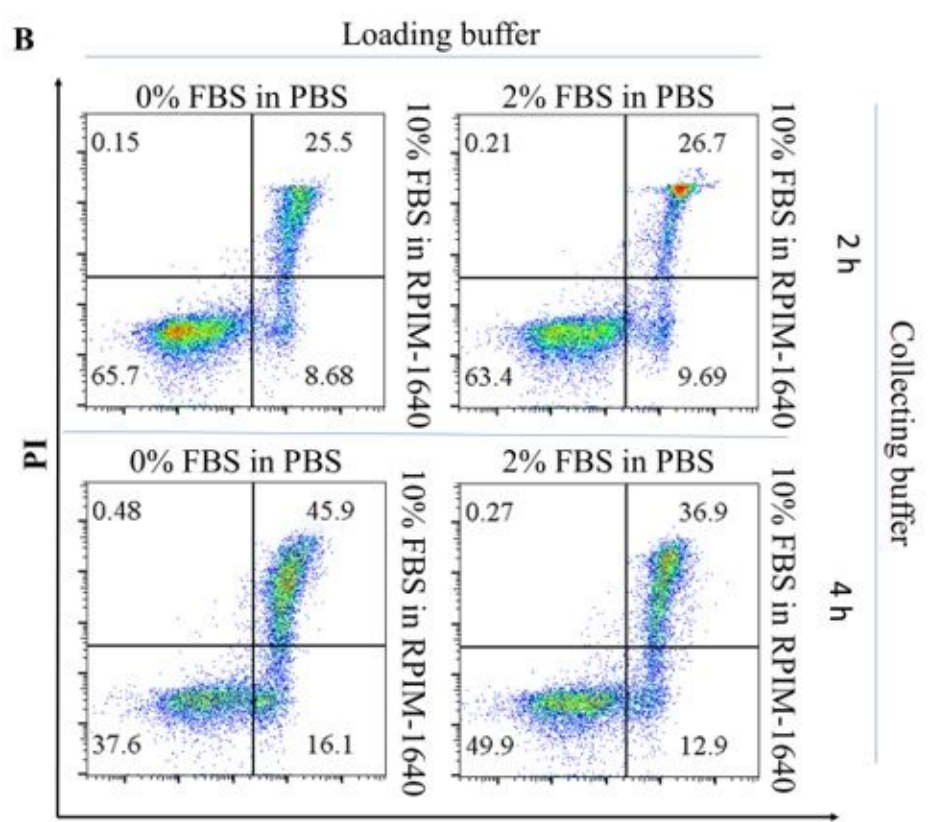

Annexin V - FITC

D

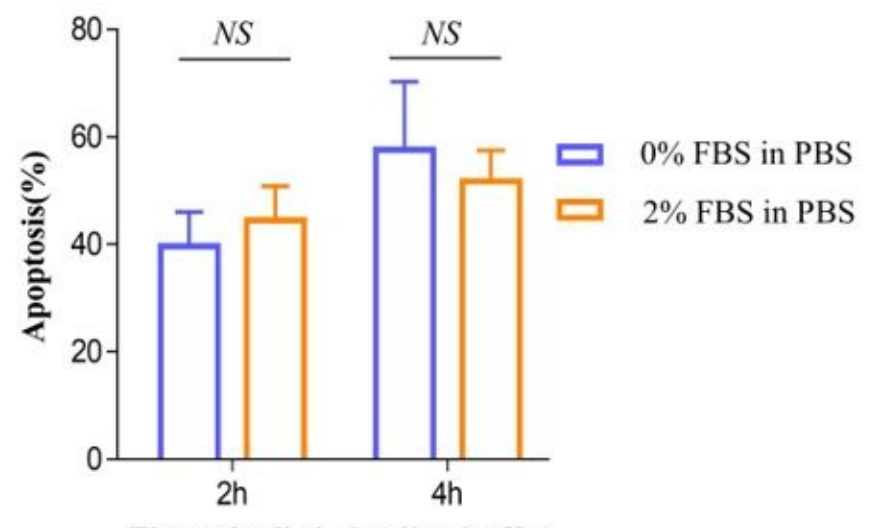

Time of cells in loading buffer

\section{Figure 2}

Effects of the addition of FBS in loading buffer on cell apoptosis. A. Dot plots of apoptotic cells sorted with different loading and collecting buffers. B. Dot plots of apoptotic cells sorted with different loading and collecting buffers after 2 hours and 4 hours. Numbers in quadrants indicate the percentages of cells in each throughout. Cells were stained with Annexin- $V$ and propidium iodide and analyzed by flow cytometry. C-D. The data of the percentage of apoptotic cells of three independent experiments. Data are presented as the mean \pm SEM. Each experiment was repeated at least 3 times. 

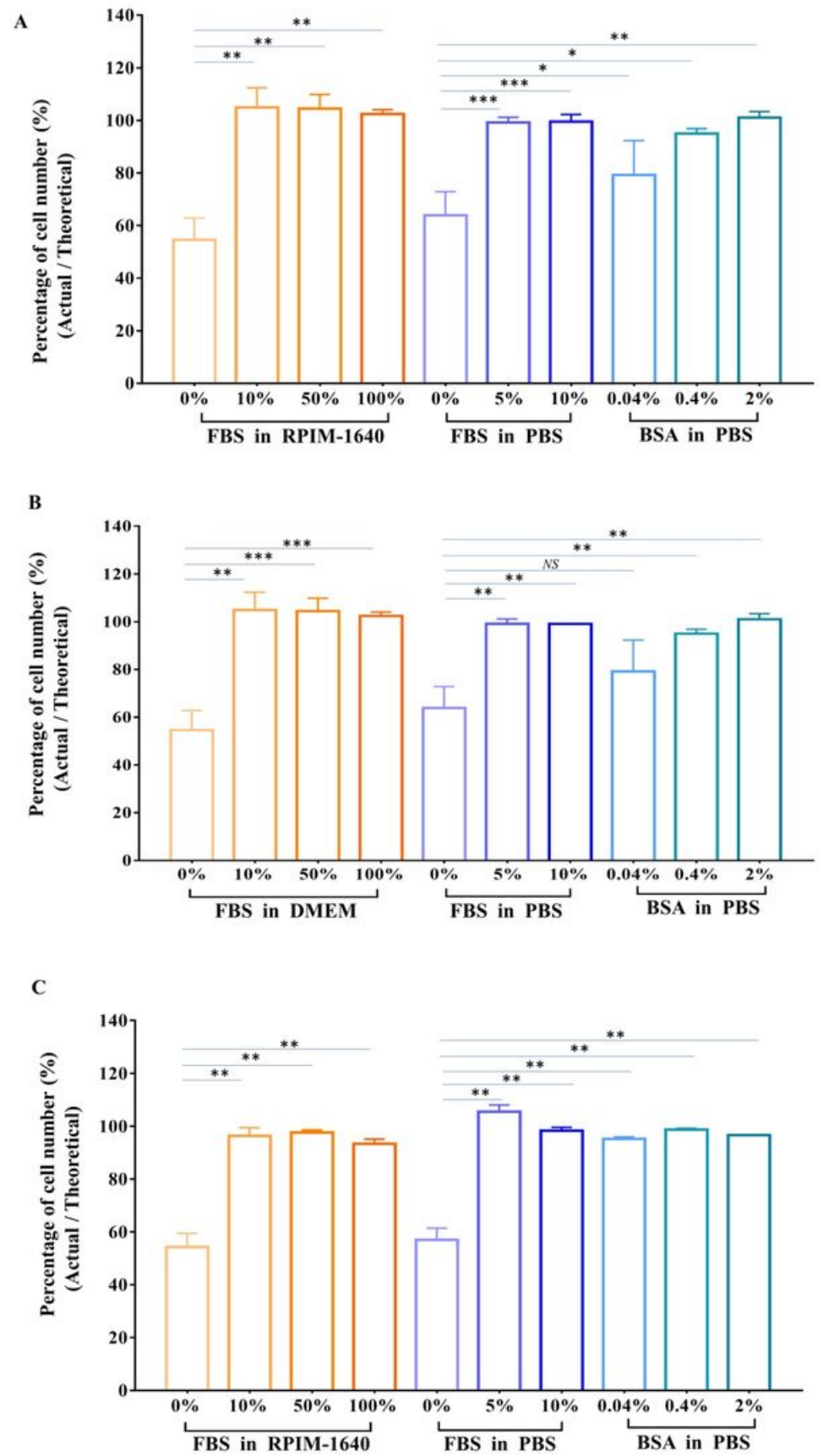

\section{Figure 3}

Effects of the additions of FBS/BSA in collecting buffer on cell counts. A is Jurkat cells. B shows 293T cells. C. shows CD45+ cells from mouse spleen. Cell counts for sorted cells using different collecting buffers. We collected 500,000 cells using each collecting buffer. A $50 \mu$ cell suspension was tested by flow cytometry. The actual cell amounts were counted. Based on the total cell amounts and weight difference before and after sorting to estimate the theoretical cell amounts in $50 \mu \mathrm{l}$ collecting buffer, the recovery rate was obtained by the actual cell amounts/the theoretical cell amounts. Data are presented as the mean $\pm \mathrm{SEM}$. Each experiment was repeated at least 3 times. ${ }^{\star} p<0.05,{ }^{*} p<0.01,{ }^{* \star} p<0.001$, by one-way ANOVA. 

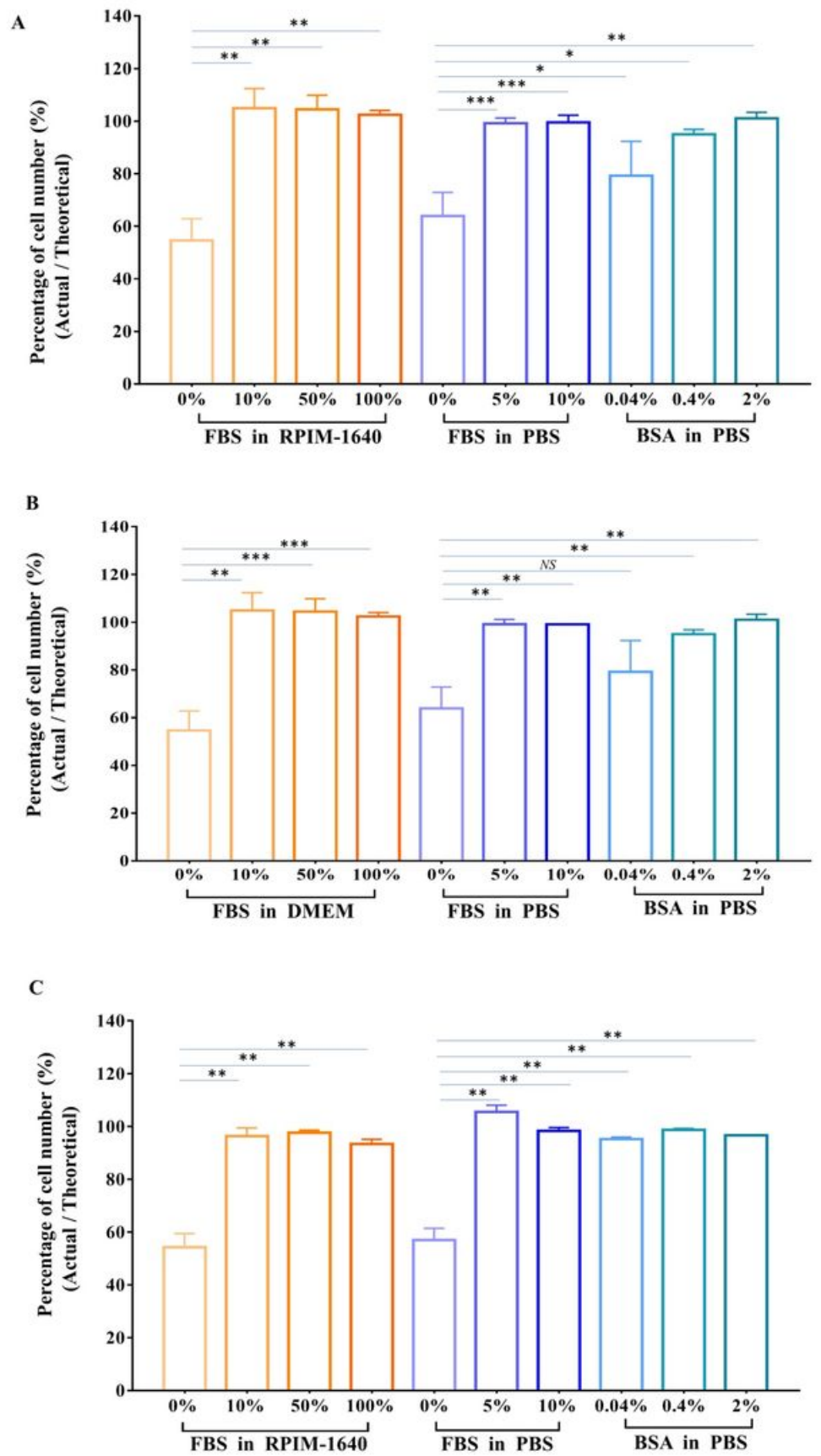

\section{Figure 3}

Effects of the additions of FBS/BSA in collecting buffer on cell counts. A is Jurkat cells. B shows 293T cells. C. shows CD45+ cells from mouse spleen. Cell counts for sorted cells using different collecting buffers. We collected 500,000 cells using each collecting buffer. A $50 \mu$ cell suspension was tested by flow cytometry. The actual cell amounts were counted. Based on the total cell amounts and weight difference before and after sorting to estimate the theoretical cell amounts in $50 \mu \mathrm{l}$ collecting buffer, the recovery rate was obtained by the actual cell amounts/the theoretical cell amounts. Data are presented as the mean $\pm \mathrm{SEM}$. Each experiment was repeated at least 3 times. ${ }^{*} p<0.05,{ }^{*} p<<0.01,{ }^{* *} p<0.001$, by one-way ANOVA. 
A

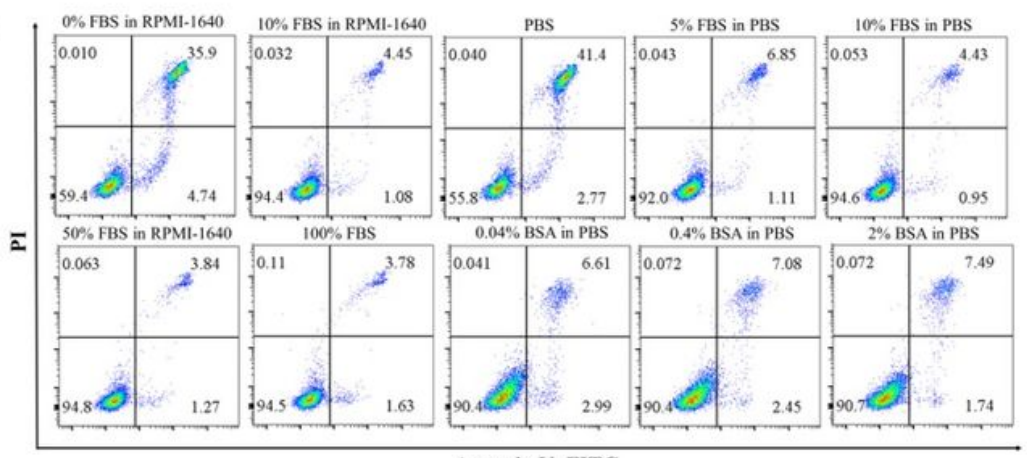

Annexin V- FITC

B

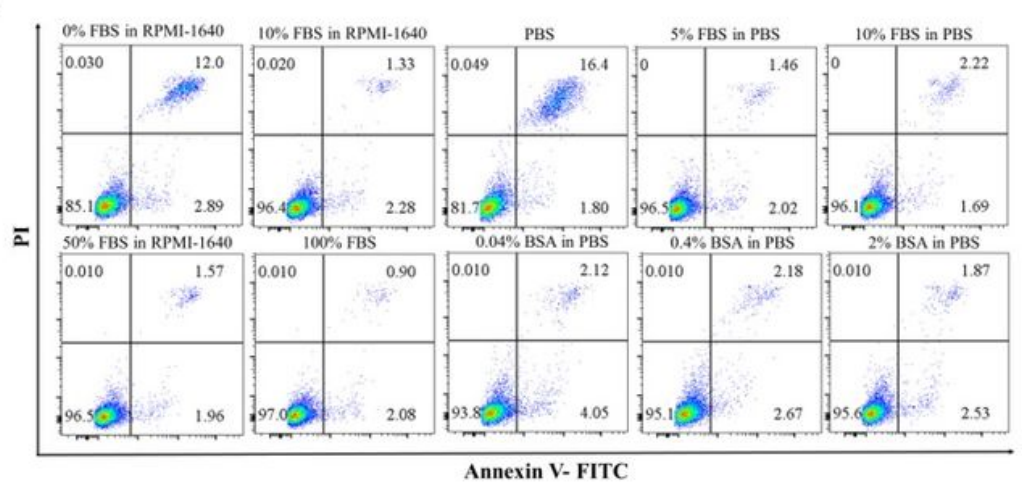

c

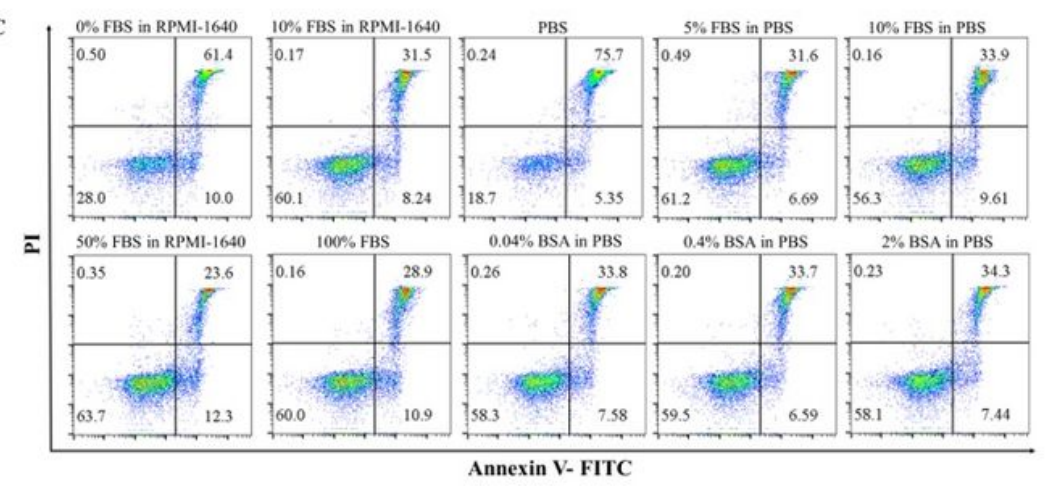

D

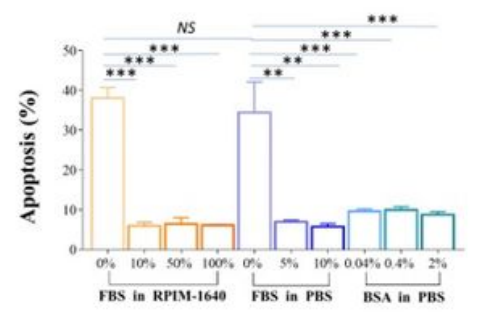

E

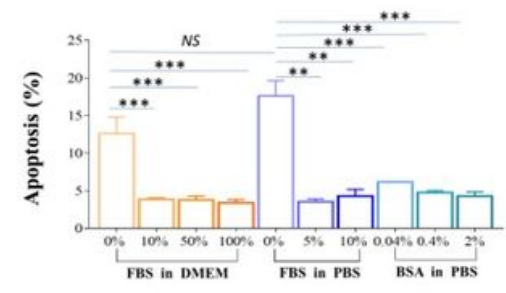

F

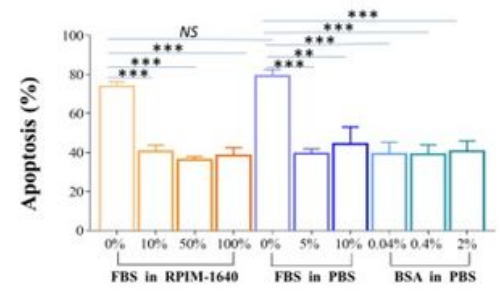

\section{Figure 4}

Effects of the addition of FBS/BSA in collecting buffer on cell apoptosis. A - C. Dot plots of apoptotic cells sorted with different collecting buffers. Numbers in quadrants indicate the percentages of cells in each throughout. Cells were stained with Annexin-V and propidium iodide and analyzed by flow cytometry. A is Jurkat cells, B is 293T cells, C is CD45+ cells from mouse spleen. D- F. The concentration of FBS or BSA in the collection buffer affects the apoptotic rate. The data of the percentage of apoptotic cells shown in D to $F$ are the means of three independent experiments. D is Jurkat cells, $E$ is 293T cells, F is CD45+ cells from mouse spleen. Data are presented as the mean \pm SEM. ${ }^{\star} p<0.05,{ }^{\star \star} p<0.01,{ }^{\star \star \star} p<0.001$, by one-way ANOVA. 
A

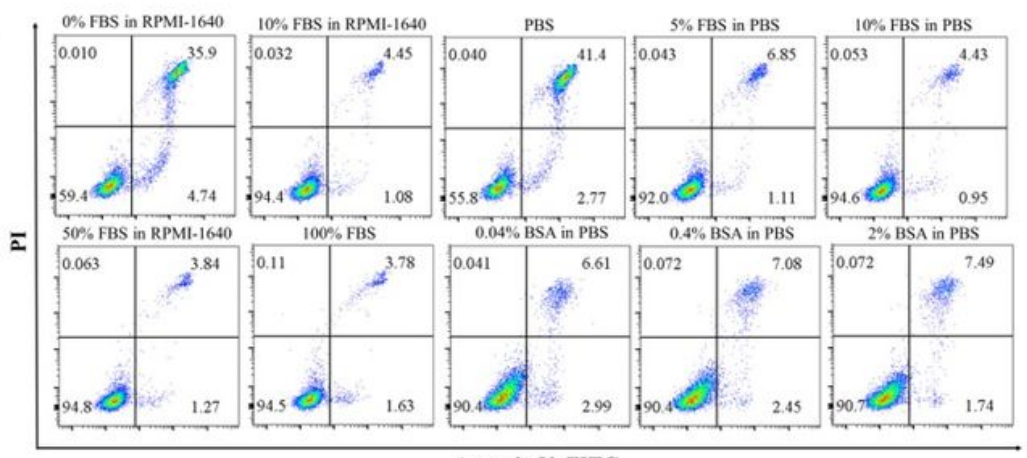

Annexin V- FITC

B

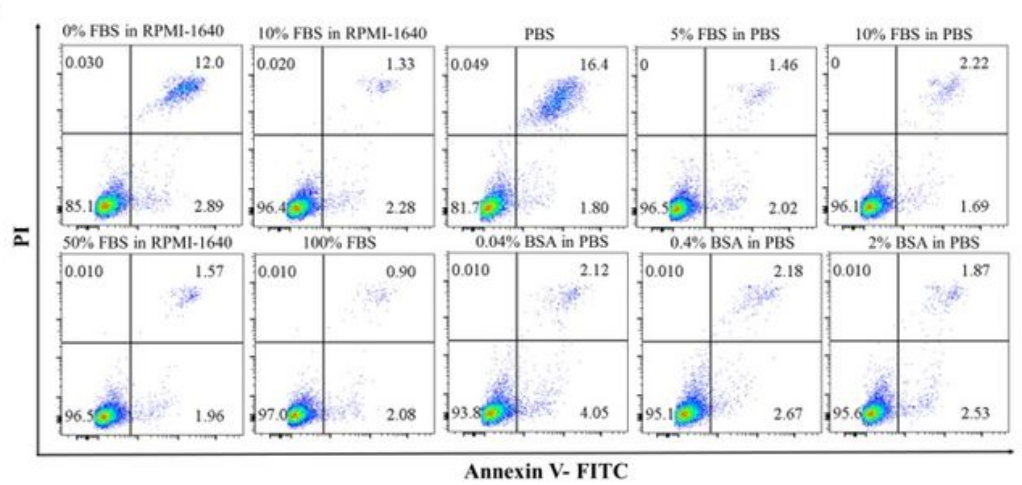

c

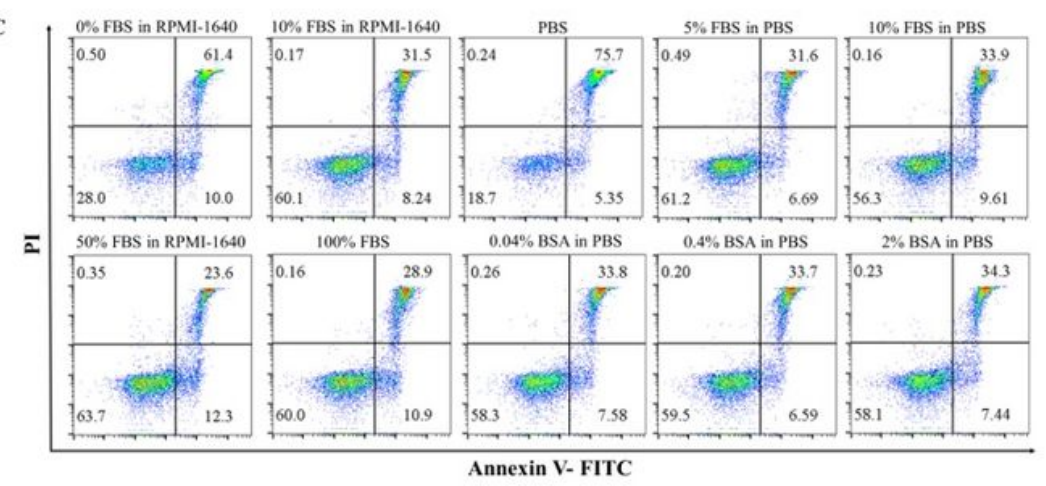

D

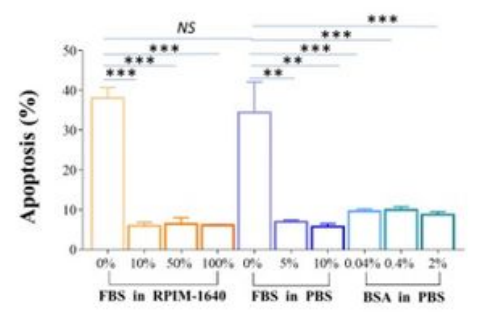

E

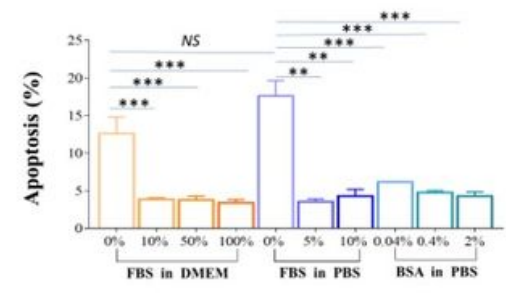

F

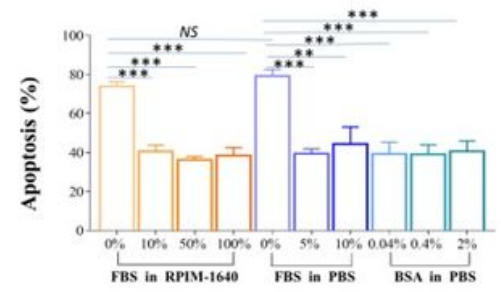

\section{Figure 4}

Effects of the addition of FBS/BSA in collecting buffer on cell apoptosis. A - C. Dot plots of apoptotic cells sorted with different collecting buffers. Numbers in quadrants indicate the percentages of cells in each throughout. Cells were stained with Annexin-V and propidium iodide and analyzed by flow cytometry. A is Jurkat cells, B is 293T cells, C is CD45+ cells from mouse spleen. D- F. The concentration of FBS or BSA in the collection buffer affects the apoptotic rate. The data of the percentage of apoptotic cells shown in D to F are the means of three independent experiments. D is Jurkat cells, $E$ is 293T cells, F is CD45+ cells from mouse spleen. Data are presented as the mean \pm SEM. ${ }^{\star} p<0.05,{ }^{\star \star} p<0.01,{ }^{\star \star \star} p<0.001$, by one-way ANOVA. 


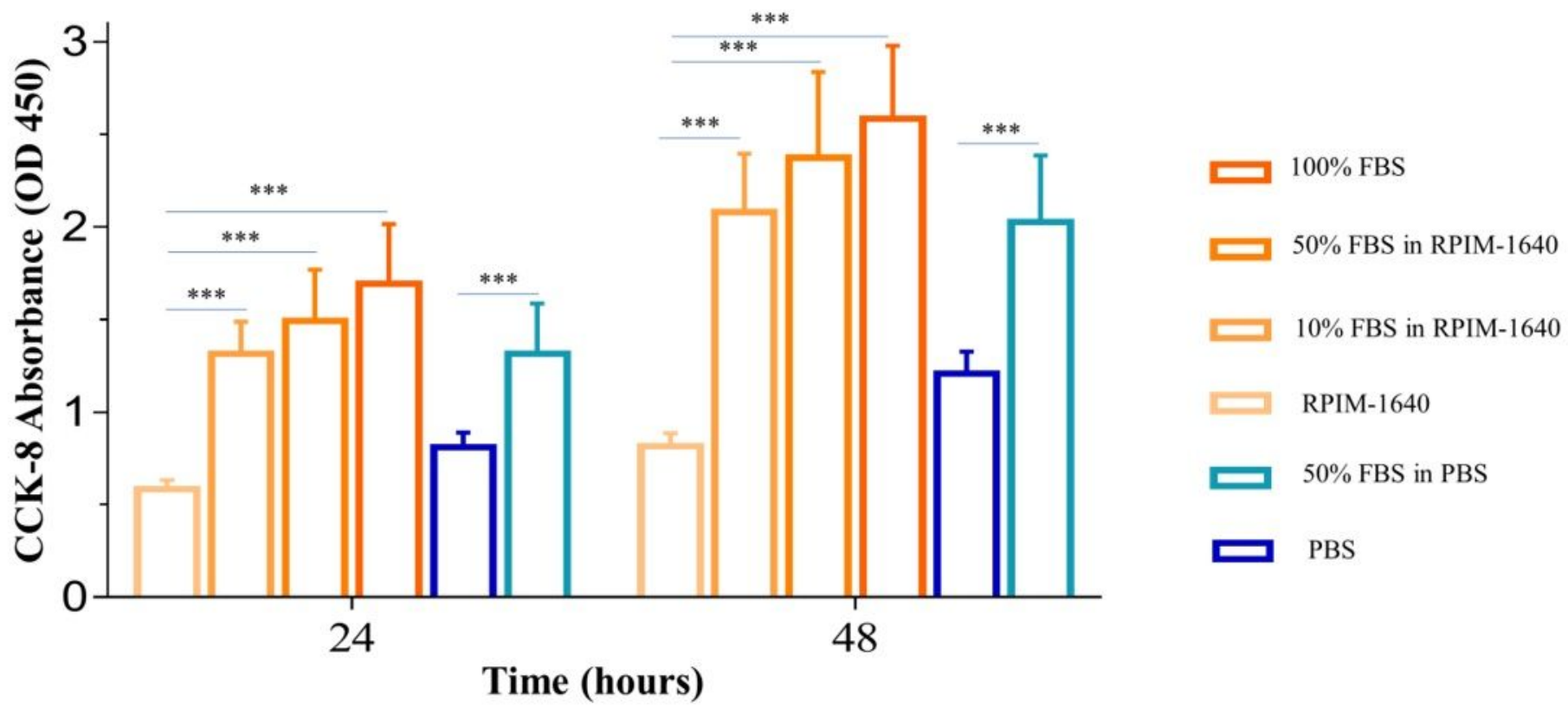

Figure 5

Effects of the addition of FBS/BSA in collecting buffer on cell proliferation. Each collecting solution contained 2,000 cells in each well. Cells were incubated for $24 \mathrm{~h}$ and $48 \mathrm{~h}$. Cell proliferation was assessed by CCK-8 assay. ${ }^{* \star *} \mathrm{P}<0.001$, as determined by two-way ANOVA.

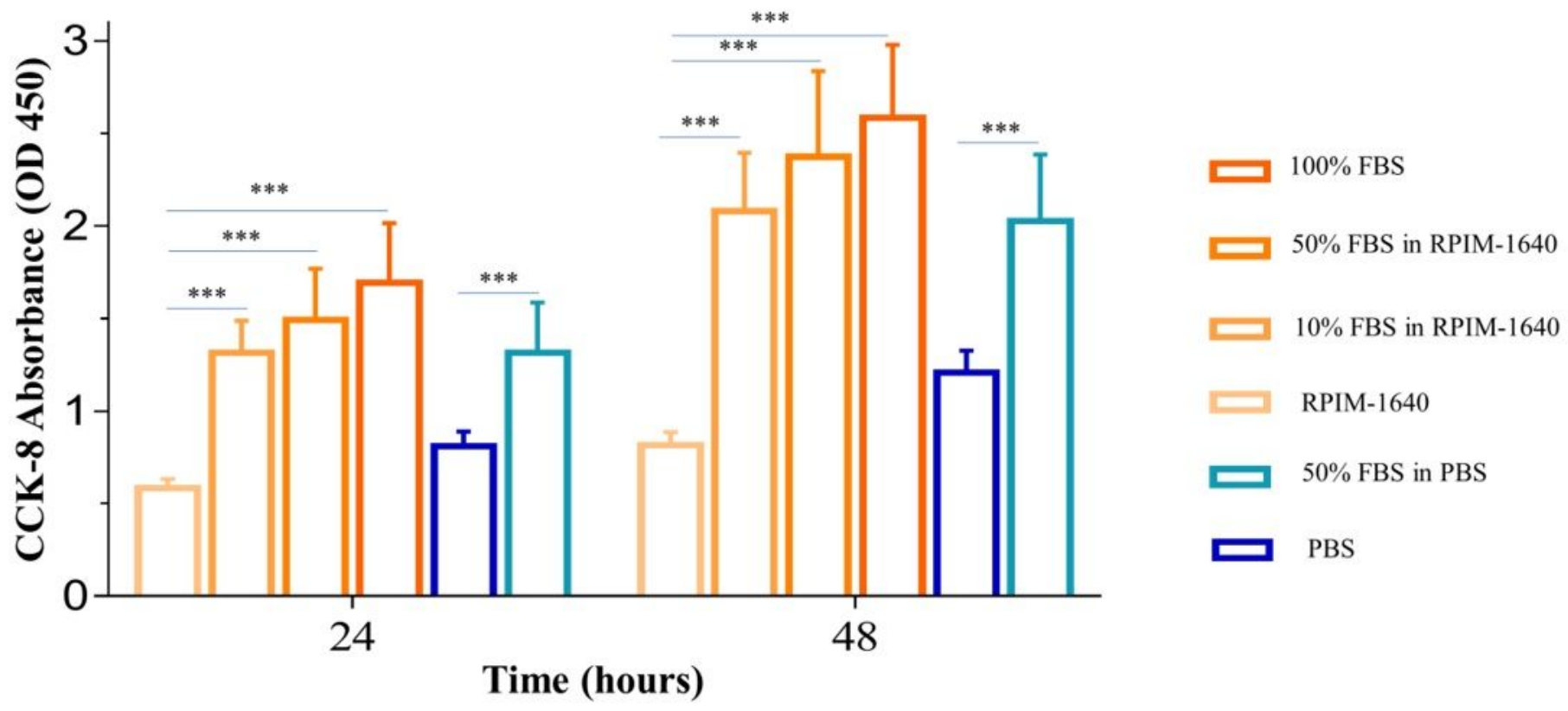

Figure 5

Effects of the addition of FBS/BSA in collecting buffer on cell proliferation. Each collecting solution contained 2,000 cells in each well. Cells were incubated for $24 \mathrm{~h}$ and $48 \mathrm{~h}$. Cell proliferation was assessed by CCK-8 
A

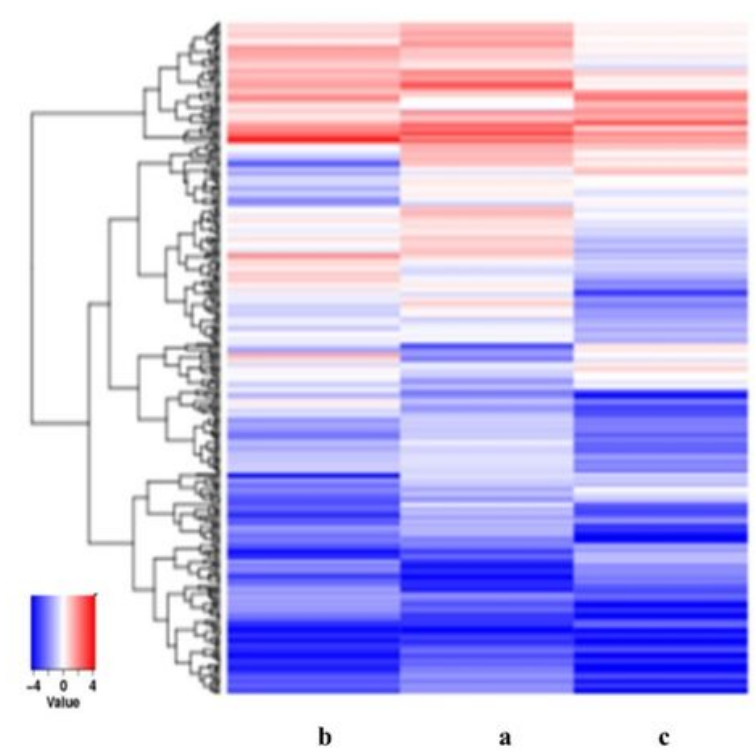

B

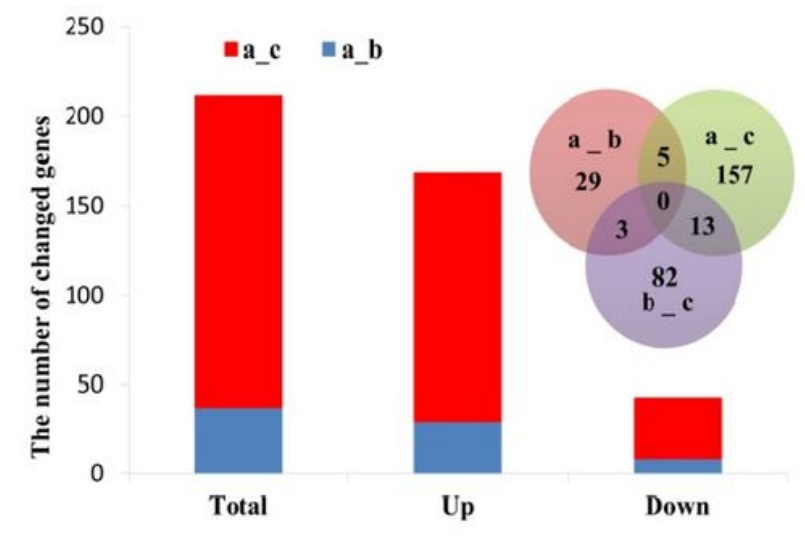

C

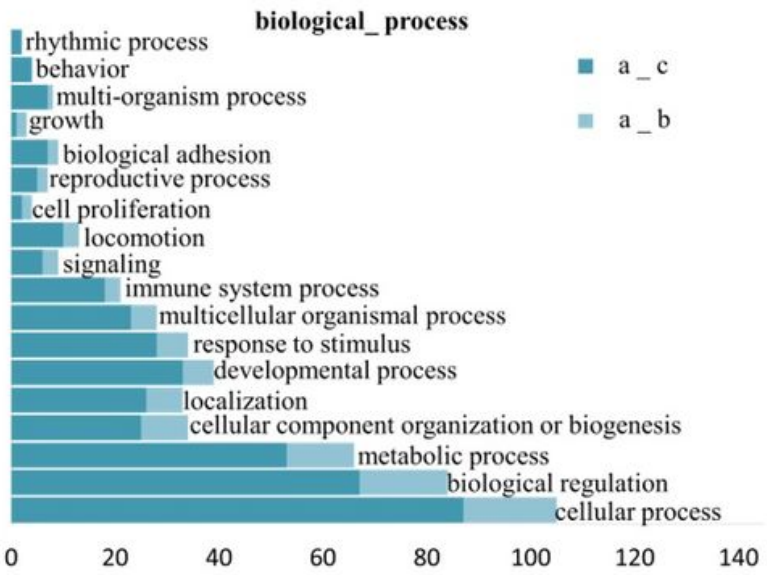

D

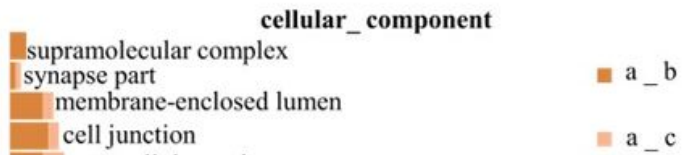

extracellular region

extracellular region part
macromolecular complex
organelle part

organelle part
membrane

membrane part

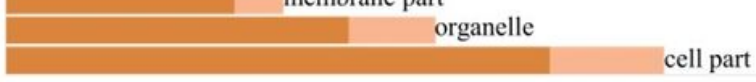

$\begin{array}{llllllll}0 & 20 & 40 & 60 & 80 & 100 & 120 & 140\end{array}$

E

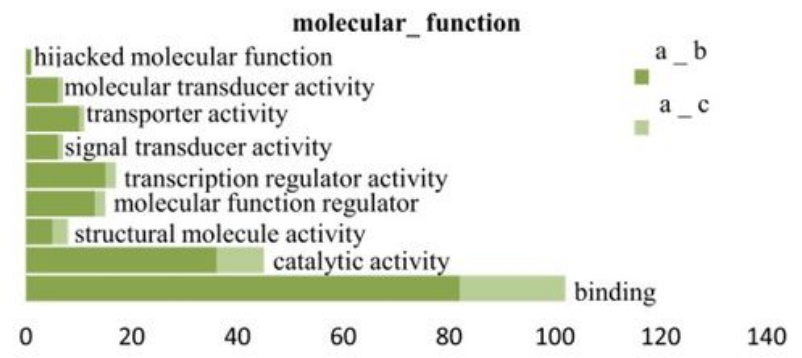

\section{Figure 6}

Effects of FBS in collecting buffer on mRNA expression in Jurkat cells. A. Hierarchical clustering analysis of unsorted cells and sorted cells were harvested using collecting buffer with or without FBS. The expression levels of genes (fold change $>2.0, \mathrm{P}<0.05$ ) are presented in different colors, indicating expression levels above and below the median expression level across all samples. B. The number of genes that were up- or downregulated of cells collected using PBS or 5\% FBS in PBS compared to unsorted cells. Venn diagram showed the overlap of genes with abnormal expression using different collecting buffers. C - E. GO annotations of affected genes from different collecting buffers. The bar plot presents the numbers of affected genes of significantly enriched GO terms in biological processes, cellular components and molecular functions. a: unsorted cells; b: sorted cells collected using 5\% FBS in PBS; c: sorted cells collected using PBS 
A

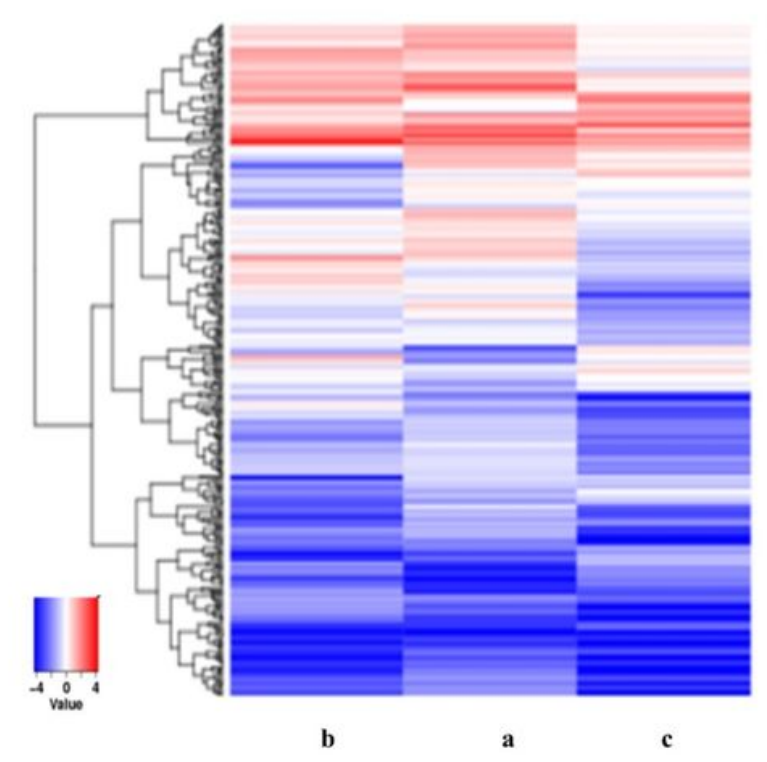

B

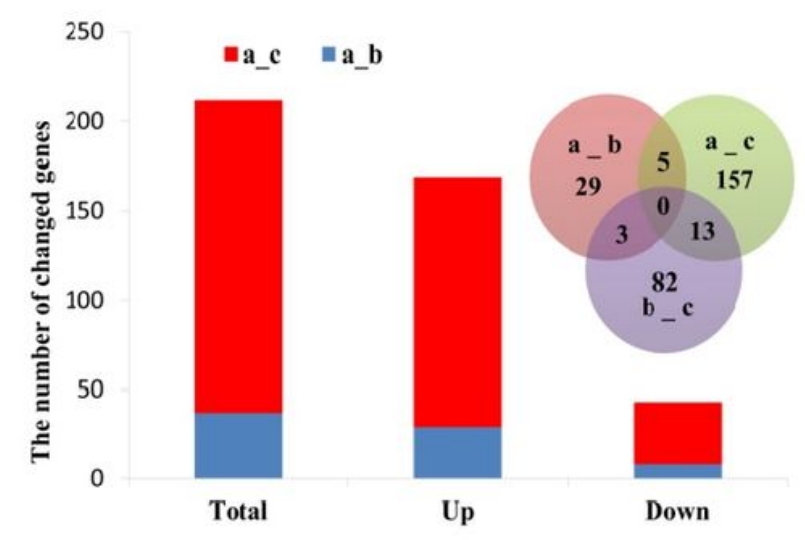

C

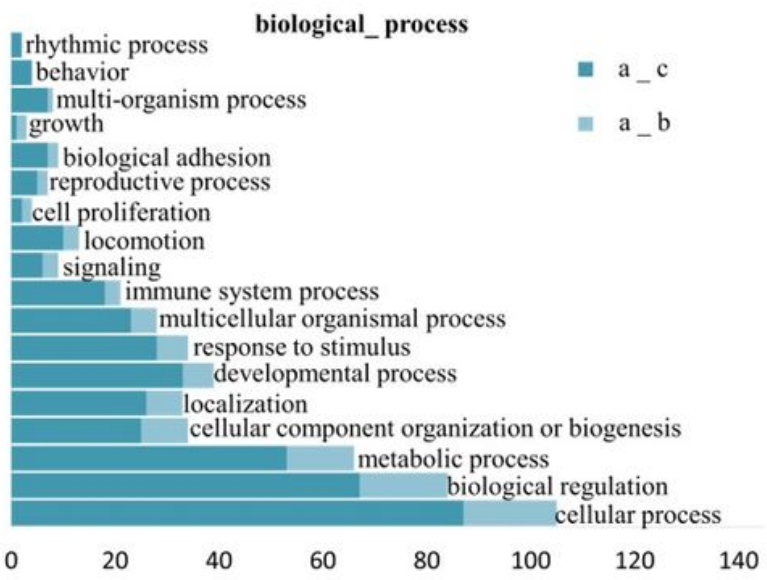

D

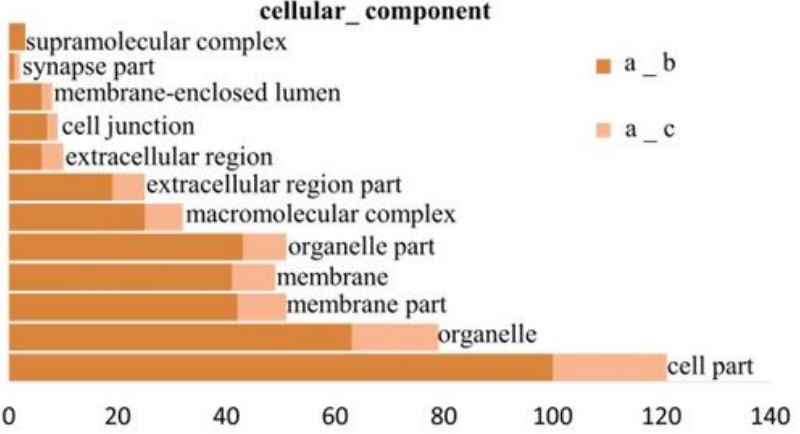

$\mathbf{E}$

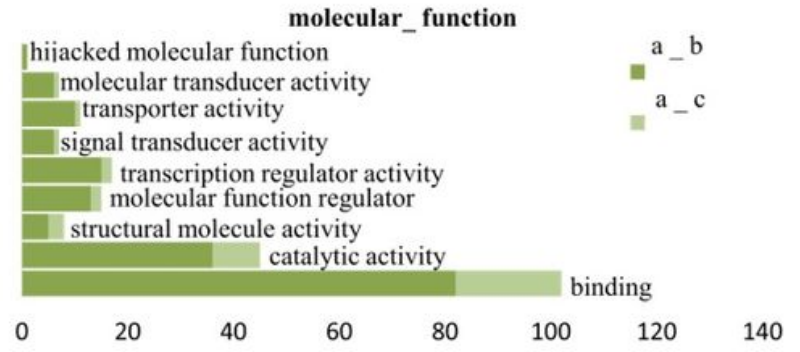

\section{Figure 6}

Effects of FBS in collecting buffer on mRNA expression in Jurkat cells. A. Hierarchical clustering analysis of unsorted cells and sorted cells were harvested using collecting buffer with or without FBS. The expression levels of genes (fold change $>2.0, P<0.05$ ) are presented in different colors, indicating expression levels above and below the median expression level across all samples. B. The number of genes that were up- or downregulated of cells collected using PBS or 5\% FBS in PBS compared to unsorted cells. Venn diagram showed the overlap of genes with abnormal expression using different collecting buffers. C - E. GO annotations of affected genes from different collecting buffers. The bar plot presents the numbers of affected genes of significantly enriched GO terms in biological processes, cellular components and molecular functions. a: unsorted cells; b: sorted cells collected using 5\% FBS in PBS; c: sorted cells collected using PBS 


\section{Three-step strategy for sorting single cells by FACS}

High yield

High viability and stable gene expression

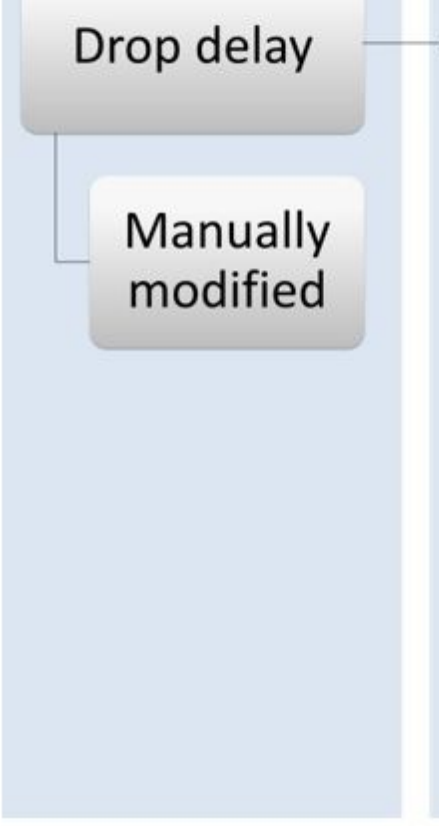

loading buffer

PBS
Collection buffer

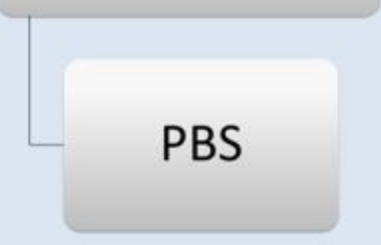

$2 \% \mathrm{BSA}$ in PBS

$10 \%$ FBS in Culture medium

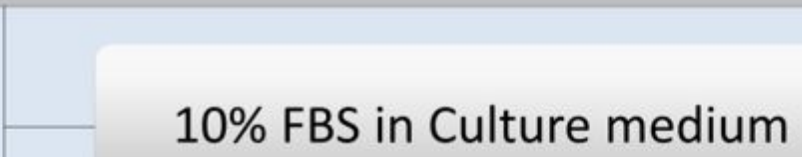

$5 \%$ FBS in PBS

\section{Figure 7}

Three-step strategy for sorting single cells by FACS. First step: Set the drop delay by manually modifying the model to obtain a high yield. Second step: PBS could be used as loading buffer, which has no significant effect on cell viability in 4 hours. Third step $₫ 10 \%$ FBS in culture medium, $2 \%$ BSA in PBS or $5 \%$ FBS in PBS could be selected as the collecting buffer depending on the different requirements of the experiment. Specifically, $5 \%$ FBS in PBS is optimal for single-cell RNA sequencing because it affects gene expression less than PBS alone. 


\section{Three-step strategy for sorting single cells by FACS}

High yield

High viability and stable gene expression

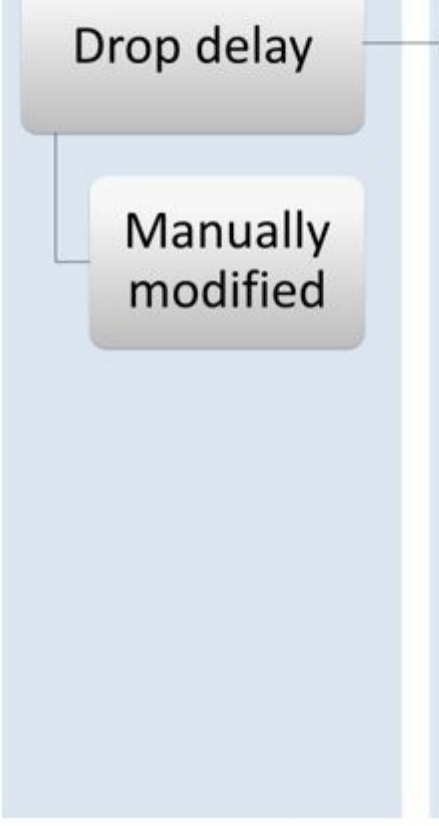

loading buffer

PBS
Collection buffer

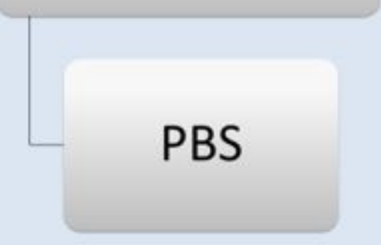

$2 \% \mathrm{BSA}$ in PBS

$10 \%$ FBS in Culture medium

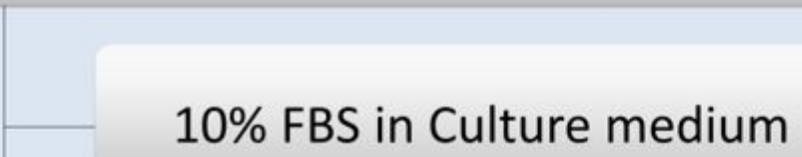

$5 \%$ FBS in PBS

\section{Figure 7}

Three-step strategy for sorting single cells by FACS. First step: Set the drop delay by manually modifying the model to obtain a high yield. Second step: PBS could be used as loading buffer, which has no significant effect on cell viability in 4 hours. Third step $10 \%$ FBS in culture medium, $2 \%$ BSA in PBS or $5 \%$ FBS in PBS could be selected as the collecting buffer depending on the different requirements of the experiment. Specifically, $5 \%$ FBS in PBS is optimal for single-cell RNA sequencing because it affects gene expression less than PBS alone. 